\title{
Hot halo gas in the Virgo cluster galaxy NGC 4569
}

\author{
D. Tschöke ${ }^{1}$, D. J. Bomans ${ }^{2}$, G. Hensler ${ }^{1}$, and N. Junkes ${ }^{3}$ \\ 1 Institut für Theoretische Physik und Astrophysik, Universität Kiel, 24098 Kiel, Germany \\ 2 Astronomisches Institut, Ruhr-Universität Bochum, Universitätsstr. 150, 44780 Bochum, Germany \\ 3 Max-Planck-Institut für Radioastronomie, Auf dem Hügel 69, 53121 Bonn, Germany
}

Received 2 February 2001 / Accepted 20 September 2001

\begin{abstract}
We have observed the Virgo cluster spiral NGC 4569 in X-rays with ROSAT $(0.1-2.4 \mathrm{keV})$ and in the optical. From the PSPC image one can distinguish different components, like the dominant source in the nuclear region, the galactic disk, and a diffuse soft component extended to the west. This latter one coincides with a giant $\mathrm{H} \alpha$ structure. In both spectral ranges the structure reaches up to $9 \mathrm{kpc}$ out of the disk. This coincidence, the soft X-ray energy distribution, and the existence of a central starburst in NGC 4569 let us conclude that the X-ray gas traces a large scale outflow from accumulating supernova explosions and stellar winds in the galactic center. The resulting physical properties of this X-ray halo are comparable to those derived from X-ray halos in edge-on galaxies, like e.g. NGC 253. We also discuss the influence of the intracluster medium on the observed X-ray and $\mathrm{H} \alpha$ morphology. The spectral $0.1-2.4 \mathrm{keV}$ distribution of the central source and the X-ray-to-H $\alpha$ luminosity ratio favour a supermassive star cluster at the very compact core rather than an accretion-powered active nucleus in agreement with the absence of a hard compact X-ray source in the ASCA band. The nearby Magellanic dwarf galaxy IC 3583 at a projected distance of only $30 \mathrm{kpc}$ reveals an unresolved X-ray point source, several blue knots in the optical, and a narrow $\mathrm{H} \alpha$ spur, pointing toward NGC 4569, detected also in the $B$ band image. This is an indication for ongoing star formation also in IC 3583. Some interaction with NGC 4569 will be discussed although the relative radial velocity between both objects of about $1300 \mathrm{~km} \mathrm{~s}^{-1}$ makes it rather unlikely.
\end{abstract}

Key words. galaxies: active - galaxies: starburst - galaxies: ISM - galaxies: individual: NGC 4569, IC 3583 X-rays: galaxies

\section{Introduction}

Since starburst activities, i.e. short epochs in the life of galaxies with exceedingly high star formation rate (SFR) compared to the averaged one, must have played an important role in the evolutionary course of most galaxies, increasing attention is drawn to understand the different mechanisms that produce such starbursts. Signatures of star formation are perceptible all over the whole electromagnetic spectral range. In the recent past we have e.g. investigated a number of galaxies in X-rays with clear attributes of nuclear starbursts: NGC 1808 (Junkes et al. 1995), NGC 2903 (Junkes \& Hensler 1996; Tschöke et al. 2001), NGC 4410 (Tschöke et al. 1999), and NGC 4303 (Tschöke et al. 2000). The first two of these objects have so-called hot-spot regions within $1 \mathrm{kpc}$ in the center, which can be identified with tracers of star formation like HıI regions in the optical and supernova remnants (SNRs) or superbubbles in the radio regime. The situation for the first two above-mentioned objects in our sample differs from galaxies like NGC 4410, NGC 4303, and NGC 4569 since

Send offprint requests to: G. Hensler,

e-mail: hensler@astrophysik. uni-kiel.de one cannot simply distinguish between nuclear starburst activity and an active galactic nucleus (AGN) for these three. The close galaxy pair NGC 4410a/b is strongly influenced by their interaction, causing central activity. A major difficulty for this system is the large distance of about $100 \mathrm{Mpc}$, resulting in resolution problems. But even for the more nearby galaxies NGC 4303 and NGC 4569 the nature of their nuclei leaves open questions. Central starbursts can e.g. be fueled by dissipative gas accumulation in a barred potential (see e.g. NGC 2903, NGC 4303). In addition to the external influence by close encounters, interaction with the cluster gas can obviously enhance the star formation activity.

Hydrodynamical simulations predict large extraplanar X-ray emitting outflows of hot gas from central and circumnuclear starbursts in giant gas-rich spirals (Suchkov et al. 1994; Strickland et al. 2000). Also AGNs can produce central outflows, but these differ from the former ones in the sense that they are produced by the central accretion mechanism and collimated to jets by magnetic fields. Starburst-induced gaseous outflows, also called galactic superwinds, receive their energy from stellar winds of massive young stars and accumulating supernova 
explosions, driving hot gas into the interstellar medium (ISM). Because of the density stratification in galactic disks the resulting superbubble expands mainly towards the steepest gradient, i.e. perpendicular to the disk. In its "snowplow" phase the bubble sweeps up and shock-heats the ambient ISM. Depending on the strength of the starburst and the fraction of kinetic energy in the outflow, the hot gas breaks out of the dense ISM and propagates into the galactic halo (Norman \& Ikeuchi 1989; Heckman et al. 1993). If the cooling timescale takes longer than the dynamical one, the hot gas disperses in the halo and can be observed by X-rays in edge-on galaxies (Dahlem 1997). The low densities and the problem to distinguish between $\mathrm{X}$-rays from above the disk and within it make a detection of X-ray halos around galaxies with intermediate or low inclination very difficult.

As one candidate for an observed X-ray halo at intermediate inclination here we present NGC 4569, a bright early-type spiral galaxy (type Sab; Tully 1988) located within the inner $2^{\circ}$ of the Virgo cluster. It is one of the very few galaxies outside the local group with a negative radial velocity ( $\sim 250 \mathrm{~km} \mathrm{~s}^{-1}$; Stauffer et al. 1986). Its low content of Hi gas makes it difficult to determine correctly the distance by using the Tully-Fisher relation. According to Binggeli et al. (1985) and Stauffer et al. (1986) it is classified as cluster member. Hence, in the whole paper we use a distance of $16.8 \mathrm{Mpc}$ (Tully 1988; $H_{0}=75 \mathrm{~km} \mathrm{~s}^{-1}$ ), which is also confirmed by Cepheid distance measurements to the Virgo cluster (Freedman 1996). Thus 1" corresponds to 81 pc. NGC 4569 reveals a deficiency of Hi gas (Warmels 1988). Neutral hydrogen is only confined to the central few kpc of the disk, which is typical for spirals near the cluster center. The influence of the intracluster medium (ICM) on the Hi distribution in cluster spirals has been observed in several galaxy clusters (Virgo: Cayatte et al. 1990, 1994; Hercules: Dickey 1997; Coma: Bravo-Alfaro et al. 2000). The high velocity of $\sim 1250 \mathrm{~km} \mathrm{~s}^{-1}$ relative to the Virgo ICM at $1000 \mathrm{~km} \mathrm{~s}^{-1}$ (Yasuda et al. 1997) leads to the presumption that NGC 4569 is close to its pericenter in the cluster potential, just passing the center of the Virgo cluster. Consequently, the interaction with the ICM causes the ISM to be removed from the galactic edge by ram pressure stripping (e.g. Balsara et al. 1994). Furthermore, NGC 4569 is located within the observable central X-ray cooling flow of the Virgo cluster (Böhringer et al. 1994). We will discuss the effect of the interaction between the Virgo ICM and NGC 4569 and its consequences on the observed structure in Sect. 5.5.

An extremely compact nucleus in NGC 4569 was found by Maoz et al. (1995) but has not even been resolved by HST FOC observations in the UV, resulting in an upper limit of $0.05^{\prime \prime}$ or $4 \mathrm{pc}$ for its diameter in the HST F220W filter (Pogge et al. 2000). In contrast Barth et al. (1998) and Barth \& Shields (2000) report a clear extension of the nuclear source with an $F W H M$ of $13 \times 9$ pc and $14 \times 8$ pc at $2200 \AA$ and in the $V$ band HST image, respectively. The authors explain these different results to originate from aberrated PSF and saturation effects in the data used by Maoz et al. (1995). Earlier optical observations of this unique compact nucleus led to its interpretation as a LINER 2, however, with some doubt because of weak [OI] and [SII] lines (Stauffer 1983; Keel 1983). An optical investigation by Keel (1996) compares starburst and AGN features of the nucleus of NGC 4569 and explains the target as a very compact starburst dominated by supergiant stars. This stellar cluster is as large as the core of 30 Dor, but more than 6 mag brighter. In a LINER sample of HST observations (Pogge et al. 2000) NGC 4569 has the brightest UV nucleus. According to Keel (1996), the interpretation as an AGN is equivocal because of constraints provided by small line widths and dominance of a single photospheric temperature in the optical spectra. ASCA observations of NGC 4569 (Terashima et al. 2000) lack of hard X-rays, which convincingly contradicts the existence of an AGN.

In a separate paper we report the detection of a giant extraplanar $\mathrm{H} \alpha$ feature up to $9 \mathrm{kpc}$ above the disk of NGC 4569 (Bomans et al. 2001). The origin of this structure is not yet fully understood. Ionization of halo gas from a central starburst region has been discussed. The present paper is dedicated to further analyses from X-ray observations, regarding the nuclear activity of NGC 4569 . The radial extension of the $\mathrm{H} \alpha$ disk is much smaller than the stellar component and coincides with the HI distribution, indicating the effect of the ICM on the ISM of NGC 4569. Moreover, several $\mathrm{H} \alpha$ emission knots are located outside the main $\mathrm{H} \alpha$ disk.

Additionally, we discuss optical broad-band and X-ray data from the nearby Magellanic irregular galaxy IC 3583 , which is located $\sim 6^{\prime}$ or in a projected distance of $30 \mathrm{kpc}$ northwest of NGC 4569. Its radial velocity of $+1022 \mathrm{~km} \mathrm{~s}^{-1}$ (Garcia 1993) places it into the regime of the Virgo cluster, too. The large difference of $\sim 1300 \mathrm{~km} \mathrm{~s}^{-1}$ in radial velocity to NGC 4569 makes a direct connection between both galaxies rather unlikely but cannot be excluded without any doubt. Garcia (1993) classified different galaxy groups in the Virgo cluster according to spherical position and radial velocity, and associates IC 3583 with the NGC 4472 group of galaxies. A lower limit of $14 \mathrm{Mpc}$ for the distance to IC 3583 is derived by Tanvir et al. (1991) from a search for supergiant stars in a high-resolution CCD image of that galaxy. Sandage \& Bedke (1985) have included this object as an excellent target in their candidate list for resolving supergiant stars in distant galaxies with the Hubble Space Telescope. Nevertheless, for IC 3583 we also adopt a distance of $d=16.8 \mathrm{Mpc}$ according to the Virgo cluster distance (Tully 1988). Some basic parameters of our target galaxies are compiled in Table 1.

The outline of this paper is as follows. In Sect. 2 we present the optical and X-ray data and the reduction process. The results on the optical and X-ray observations are given separately in Sects. 3 and 4. Section 5 contains the discussion of the results for nucleus, disk, and halo emission. The paper closes with the conclusions in Sect. 6 . 

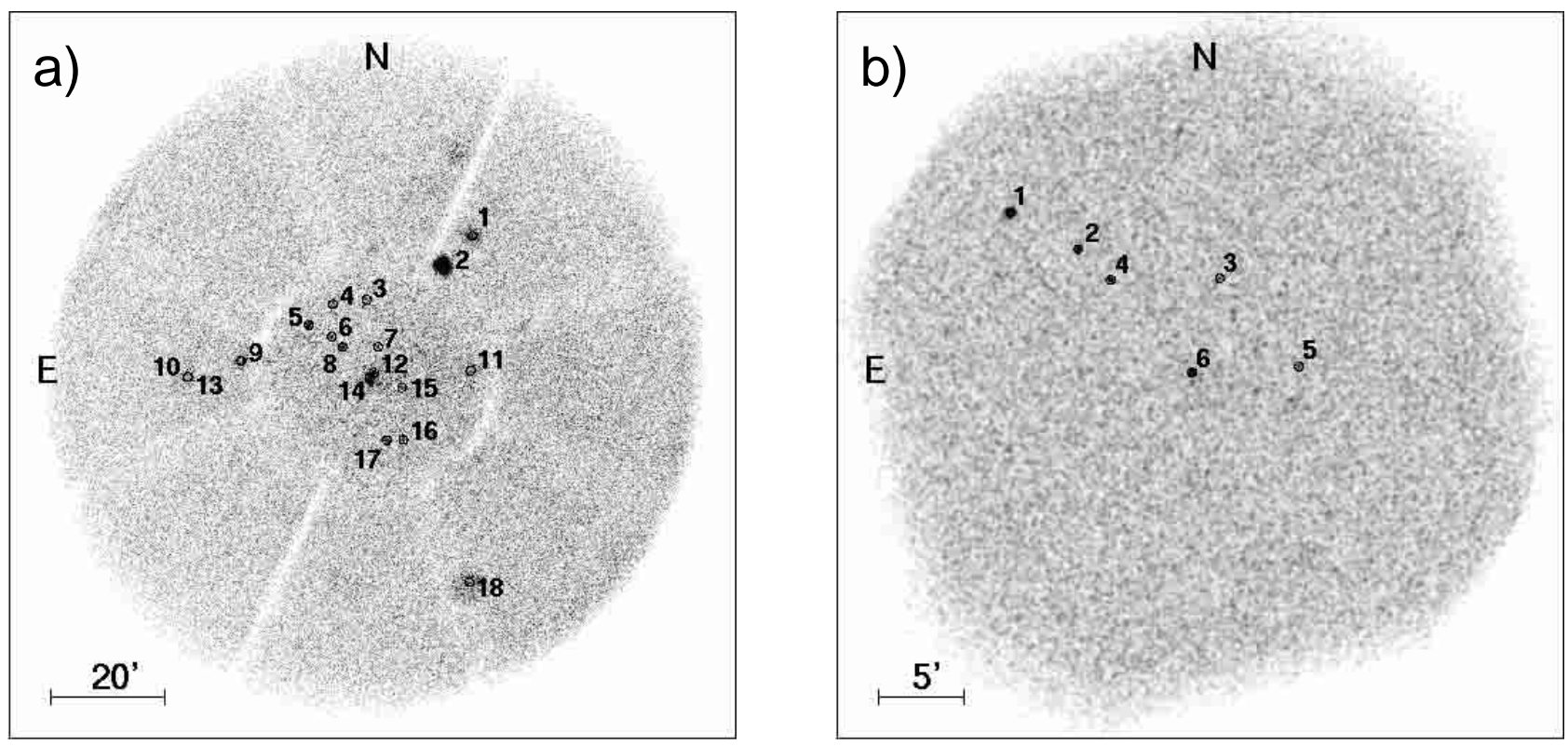

Fig. 1. Field of view from the ROSAT a) PSPC and b) HRI pointed observations of NGC 4569. All sources detected with a maximum likelihood threshold of $5 \sigma$ are plotted. The corresponding labels refer to the source numbers in Tables 3 and 4 .

Table 1. NGC 4569 and IC 3583: some basic parameters.

\begin{tabular}{lccc}
\hline & NGC 4569 & IC 3583 & Ref. $^{a}$ \\
\hline alternate name & M 90 & UGC 7784 & \\
type & Sab & Irr & 1 \\
RA $(2000)$ & $12^{\mathrm{h}} 36^{\mathrm{m}} 49.8$ & $12^{\mathrm{h}} 36^{\mathrm{m}} 43^{\mathrm{s} .5}$ & 2 \\
Dec $(2000)$ & $+13^{\circ} 09^{\prime} 46^{\prime \prime}$ & $+13^{\circ} 15^{\prime} 34^{\prime \prime}$ & 2 \\
radial velocity & $-250 \mathrm{~km} \mathrm{~s}^{-1}$ & $+1022 \mathrm{~km} \mathrm{~s}^{-1}$ & 3,4 \\
distance & $16.8 \mathrm{Mpc}$ & $16.8 \mathrm{Mpc}$ & 1 \\
$D_{25}$ diameter & $8^{\prime} .8$ & 2.5 & 1 \\
axial ratio & 0.51 & 0.64 & 3 \\
inclination & $64^{\circ}$ & $55^{\circ}$ & 1 \\
$\log \left(L_{B} / L_{\odot}\right)$ & 10.70 & 9.20 & 1 \\
$\log \left(M_{\mathrm{HI}} / M_{\odot}\right)$ & 9.28 & 8.73 & 1 \\
$\log \left(N_{\mathrm{HI}} / \mathrm{cm}^{-2}\right)($ Galactic $)$ & 20.39 & 20.39 & 5 \\
$\log \left(N_{\mathrm{HI}} / \mathrm{cm}^{-2}\right)($ intrinsic $)$ & 20.71 & & 6 \\
\hline
\end{tabular}

${ }^{a}$ References:

1) Tully (1988);

2) Neff \& Hutchings (1992) (for NGC 4569 ); Falco et al. (1999) (for IC 3583);

3) Stauffer et al. (1986);

4) Garcia (1993);

5) Dickey \& Lockman (1990);

6) Cayatte et al. (1990).

\section{Observations and data reduction}

\subsection{X-ray observations}

The field around NGC 4569 was observed with the ROSAT PSPC (central coordinates: RA (2000) = $12^{\mathrm{h}} 36^{\mathrm{m}} 48^{\mathrm{s}} .0$, Dec $\left.(2000)=+13^{\circ} 10^{\prime} 12^{\prime \prime}\right)$ with total exposure time of $18 \mathrm{ksec}$ and with the HRI (central coordinates: RA $(2000)=12^{\mathrm{h}} 36^{\mathrm{m}} 48^{\mathrm{s}} .0$, Dec $(2000)=$ $+13^{\circ} 9^{\prime} 36^{\prime \prime}$ ) for a total of $24 \mathrm{ksec}$. For the observation log for both data sets see Table 2 .
The observations were analyzed using the EXSAS (Zimmermann et al. 1993), XSPEC (Arnaud 1996) and IDL software packages. All images presented in the following are normalized to values of counts per seconds and pixel. Figure 2 shows a $17^{\prime} \times 17^{\prime}$ POSS II image centered on NGC 4569 overlaid by contours of the central $17^{\prime}$ of the PSPC field (pixel size of $5^{\prime \prime}$ and convolved with a Gaussian of $25^{\prime \prime} F W H M$ ). The X-ray images have been corrected for vignetting but not for contributions of the background. They contain the total X-ray emission in the energy interval of $0.1-2.4 \mathrm{keV}$. The $5 \sigma$ sources detected in the PSPC and HRI field of view with a maximum likelihood method are numbered in Fig. 1, and listed in the Tables 3 and 4 together with optical identifications. Source No. 14 in the PSPC image coincides with NGC 4569 while source No. 7 is located at the optical IC 3583. These two sources correspond to Nos. 6 and 3 in the HRI image. Source No. 12 in Fig. 1a near NGC 4569 is probably a stellar foreground source (see Sect. 4.1.1).

The background value in the PSPC image was determined by three circular source-free fields near NGC 4569 with diameters of $164^{\prime \prime}, 158^{\prime \prime}$, and $150^{\prime \prime}$, respectively. The average background flux amounts to $6.3 \times 10^{-7}$ cts s$^{-1} \operatorname{arcsec}^{-2}$. The background flux in the energy range of $0.4-2.4 \mathrm{keV}$ for this region results in $2.6 \times 10^{-7}$ cts s$^{-1} \operatorname{arcsec}^{-2}$ for our data, in agreement with determinations by Böhringer et al. (1994). The gradient of the hot ICM is too flat to show significant changes of the background in the direct vicinity of NGC 4569 and IC 3583. Nevertheless, a very slight increase is perceptible at the western edge of the FOV (Fig. 1a) toward the Virgo cluster X-ray center near M87. The same procedure as for the PSPC data was carried out in the HRI image with the same background fields. The background for the HRI exposure was determined to be $3.7 \times 10^{-7} \mathrm{cts} \mathrm{s}^{-1} \operatorname{arcsec}^{-2}$. 
Table 2. ROSAT observations of NGC 4569 .

\begin{tabular}{lccccc}
\hline Detector & $\begin{array}{c}\text { RA } \\
(2000)\end{array}$ & $\begin{array}{c}\text { Dec } \\
(2000)\end{array}$ & Time of Obs. & $\begin{array}{c}\text { Exposure } \\
{[\mathrm{s}]}\end{array}$ & $\begin{array}{c}\text { No. of OBIs } \\
\end{array}$ \\
\hline PSPC & $12^{\mathrm{h}} 36^{\mathrm{m}} 48^{\mathrm{s}} .0$ & $+13^{\circ} 10^{\prime} 12^{\prime \prime}$ & Dec. 19-21, 1992 & 17976 & 14 \\
HRI & $12^{\mathrm{h}} 36^{\mathrm{m}} 48^{\mathrm{s}} .0$ & $+13^{\circ} 09^{\prime} 36^{\prime \prime}$ & Jul. 10-11, 1994 & 2109 & 1 \\
& $12^{\mathrm{h}} 36^{\mathrm{m}} 48^{\mathrm{s}} .0$ & $+13^{\circ} 09^{\prime} 36^{\prime \prime}$ & Jun. 18-21, 1995 & 21858 & 10 \\
\hline
\end{tabular}

Table 3. Source detections in the ROSAT PSPC observation of NGC 4569 with a maximum likelihood threshold of $14(5 \sigma)$.

\begin{tabular}{|c|c|c|c|c|c|c|}
\hline No. & RA (2000) & $\operatorname{Dec}(2000)$ & $\begin{array}{l}\text { Existence } \\
\text { likelihood }\end{array}$ & $\begin{array}{l}\text { Count rate }(\mathrm{PSPC})^{a} \\
{\left[10^{-3} \mathrm{cts} \mathrm{s}^{-1}\right]}\end{array}$ & $\begin{array}{l}\text { No. } \\
\text { (HRI) }\end{array}$ & Identification \\
\hline 1 & $12^{\mathrm{h}} 35^{\mathrm{m}} 35^{\mathrm{s}} .7$ & $+13^{\circ} 34^{\prime} 43^{\prime \prime}$ & 173 & $29.8 \pm 2.1$ & & \\
\hline 2 & $12^{\mathrm{h}} 35^{\mathrm{m}} 57^{\mathrm{s}} .3$ & $+13^{\circ} 29^{\prime} 29^{\prime \prime}$ & 7237 & $317 \pm 4$ & & $\mathrm{BD}+142519$ \\
\hline 3 & $12^{\mathrm{h}} 36^{\mathrm{m}} 51^{\mathrm{s}} .8$ & $+13^{\circ} 23^{\prime} 34^{\prime \prime}$ & 16 & $3.4 \pm 0.7$ & & QSO $1234+136$ \\
\hline 4 & $12^{\mathrm{h}} 37^{\mathrm{m}} 16^{\mathrm{s}} .2$ & $+13^{\circ} 22^{\prime} 43^{\prime \prime}$ & 23 & $4.0 \pm 0.8$ & & \\
\hline 5 & $12^{\mathrm{h}} 37^{\mathrm{m}} 33^{\mathrm{s}} .4$ & $+13^{\circ} 19^{\prime} 07^{\prime \prime}$ & 109 & $9.0 \pm 0.9$ & 1 & LBQS $1235+1335$ \\
\hline 6 & $12^{\mathrm{h}} 37^{\mathrm{m}} 17^{\mathrm{s}} 0$ & $+13^{\circ} 17^{\prime} 02^{\prime \prime}$ & 35 & $3.6 \pm 0.6$ & 2 & \\
\hline 7 & $12^{\mathrm{h}} 36^{\mathrm{m}} 43^{\mathrm{s}} .7$ & $+13^{\circ} 15^{\prime} 16^{\prime \prime}$ & 40 & $3.3 \pm 0.6$ & 3 & IC 3583 \\
\hline 8 & $12^{\mathrm{h}} 37^{\mathrm{m}} 09^{\mathrm{s}} .4$ & $+13^{\circ} 15^{\prime} 13^{\prime \prime}$ & 230 & $11.2 \pm 0.9$ & 4 & \\
\hline 9 & $12^{\mathrm{h}} 38^{\mathrm{m}} 22^{\mathrm{s}} .4$ & $+13^{\circ} 12^{\prime} 46^{\prime \prime}$ & 153 & $23.1 \pm 1.7$ & & QSO $1235+1329$ \\
\hline 10 & $12^{\mathrm{h}} 39^{\mathrm{m}} 00^{\mathrm{s}} .9$ & $+13^{\circ} 10^{\prime} 00^{\prime \prime}$ & 53 & $18.4 \pm 2.1$ & & \\
\hline 11 & $12^{\mathrm{h}} 35^{\mathrm{m}} 36^{\mathrm{s}} .9$ & $+13^{\circ} 11^{\prime} 05^{\prime \prime}$ & 20 & $9.1 \pm 1.4$ & & \\
\hline 12 & $12^{\mathrm{h}} 36^{\mathrm{m}} 46^{\mathrm{s}} 6$ & $+13^{\circ} 10^{\prime} 48^{\prime \prime}$ & 78 & $6.0 \pm 0.7$ & & NGC $4569^{b}$ \\
\hline 13 & $12^{\mathrm{h}} 39^{\mathrm{m}} 01^{\mathrm{s}} .0$ & $+13^{\circ} 09^{\prime} 59^{\prime \prime}$ & 58 & $21.2 \pm 2.3$ & & \\
\hline 14 & $12^{\mathrm{h}} 36^{\mathrm{m}} 49^{\mathrm{s}} .9$ & $+13^{\circ} 09^{\prime} 46^{\prime \prime}$ & 490 & $20.4 \pm 1.2$ & 6 & NGC 4569 \\
\hline 15 & $12^{\mathrm{h}} 36^{\mathrm{m}} 26^{\mathrm{s}} .5$ & $+13^{\circ} 08^{\prime} 10^{\prime \prime}$ & 20 & $2.4 \pm 0.5$ & & \\
\hline 16 & $12^{\mathrm{h}} 36^{\mathrm{m}} 25^{\mathrm{s}} .4$ & $+12^{\circ} 58^{\prime} 58^{\prime \prime}$ & 20 & $2.9 \pm 0.6$ & & \\
\hline 17 & $12^{\mathrm{h}} 36^{\mathrm{m}} 37^{\mathrm{s}} .3$ & $+12^{\circ} 58^{\prime} 53^{\prime \prime}$ & 40 & $4.7 \pm 0.7$ & & \\
\hline 18 & $12^{\mathrm{h}} 35^{\mathrm{m}} 38^{\mathrm{s}} .0$ & $+12^{\circ} 34^{\prime} 00^{\prime \prime}$ & 36 & $26.7 \pm 2.5$ & & NGC 4552 \\
\hline
\end{tabular}

${ }^{a}$ Background subtracted.

${ }^{b}$ Probably stellar foreground source, see text.

Table 4. Source detections in the ROSAT HRI observation of NGC 4569 with a maximum likelihood threshold of $14(5 \sigma)$.

\begin{tabular}{lllllll}
\hline No. & RA (2000) & Dec (2000) & $\begin{array}{l}\text { Existence } \\
\text { likelihood }\end{array}$ & $\begin{array}{l}\text { Count rate (HRI) } \\
{\left[10^{-3} \text { cts s }^{-1}\right]}\end{array}$ & $\begin{array}{l}\text { No. } \\
(\text { PSPC) }\end{array}$ & Identification \\
\hline 1 & $12^{\mathrm{h}} 37^{\mathrm{m}} 33^{\mathrm{s}} .46$ & $+13^{\circ} 19^{\prime} 09^{\prime \prime}$ & 155 & $7.8 \pm 0.7$ & 5 & LBQS 1235+1335 \\
2 & $12^{\mathrm{h}} 37^{\mathrm{m}} 17^{\mathrm{s}} .18$ & $+13^{\circ} 17^{\prime} 01^{\prime \prime}$ & 55 & $2.5 \pm 0.4$ & 6 & \\
3 & $12^{\mathrm{h}} 36^{\mathrm{m}} 43^{\mathrm{s}} .19$ & $+13^{\circ} 15^{\prime} 18^{\prime \prime}$ & 14 & $0.7 \pm 0.2$ & 7 & IC 3583 \\
4 & $12^{\mathrm{h}} 37^{\mathrm{m}} 09^{\mathrm{s}} .37$ & $+13^{\circ} 15^{\prime} 13^{\prime \prime}$ & 84 & $2.4 \pm 0.4$ & 8 & \\
5 & $12^{\mathrm{h}} 36^{\mathrm{m}} 24.12$ & $+13^{\circ} 10^{\prime} 09^{\prime \prime}$ & 18 & $0.7 \pm 0.2$ & & \\
6 & $12^{\mathrm{h}} 36^{\mathrm{m}} 49.78$ & $+13^{\circ} 09^{\prime} 48^{\prime \prime}$ & 162 & $4.6 \pm 0.5$ & 14 & NGC 4569 \\
\hline
\end{tabular}

${ }^{a}$ Background subtracted.

\subsection{Optical imaging}

We observed NGC 4569 and IC 3583 with the $1 \mathrm{~m}$ telescope at Mt. Laguna Observatory (California) in June 1995. Images were taken through a $\mathrm{H} \alpha+[\mathrm{N} \mathrm{II}]$ narrow band filter and Johnson $B$ and $R$ band filters. The detector was a thinned Tektronics $2048^{2}$ pixel CCD. The resulting field of view of the setup is $13^{\prime} \times 13^{\prime}$, therefore large enough to contain both NGC 4569 and IC 3583 on one image. The seeing was about $2^{\prime \prime}$ during the observations. The atmospheric transmission was slightly variable during the observations, therefore we did not attempt to perform an absolute flux calibration of these data. All images were reduced and registered using the IRAF data reduction package. Particular results from the $\mathrm{H} \alpha+[\mathrm{N} \mathrm{II}]$ exposure of NGC 4569 are discussed in a separate paper (Bomans et al. 2001). 


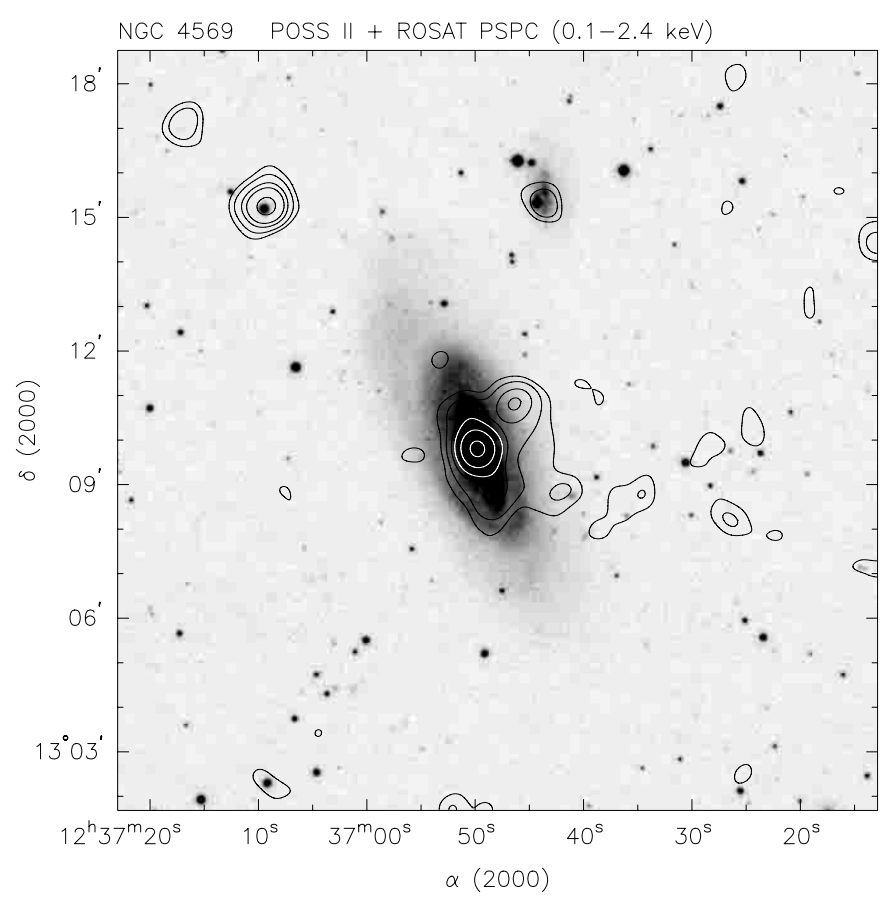

Fig. 2. PSPC observation of NGC 4569 and IC 3583 over an optical $17^{\prime} \times 17^{\prime}$ POSS II image centered on NGC 4569 .
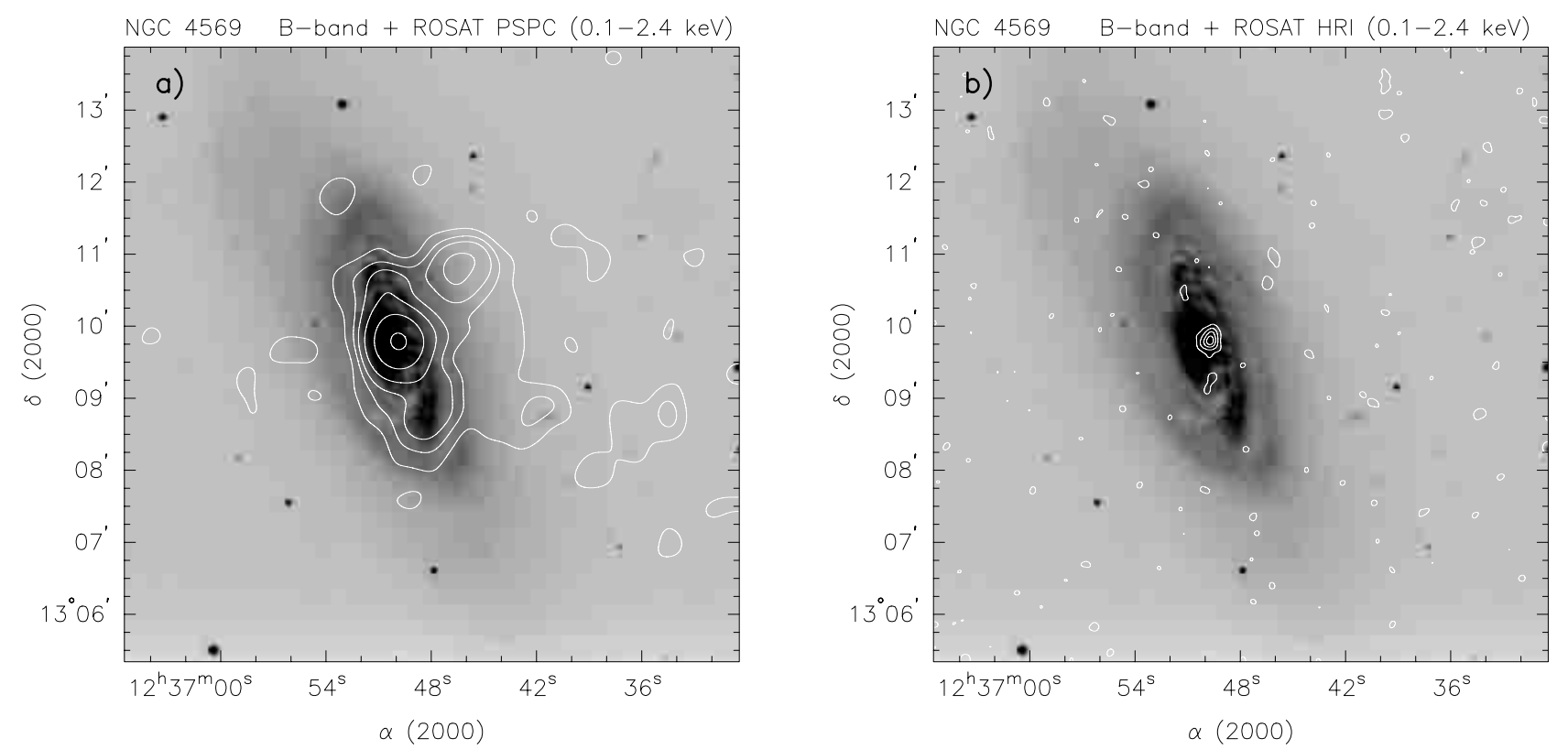

Fig. 3. The central $9^{\prime}$ of the ROSAT observation of NGC 4569 overlaid onto the $B$ band image. a) The PSPC contours represent 2, 4, 6, 10, 20 and $40 \sigma$ above the mean background of $6.5 \times 10^{-7} \mathrm{cts} \mathrm{s}^{-1} \operatorname{arcsec}^{-1}$, with $\sigma=2.0 \times 10^{-7} \mathrm{cts} \mathrm{s}^{-1} \operatorname{arcsec}^{-1}$. b) The HRI contours represent 3,10,20 and $30 \sigma$ above the mean background of $3.7 \times 10^{-7} \operatorname{cts~s}^{-1} \operatorname{arcsec}^{-1}$, with $\sigma=$ $2.3 \times 10^{-7}$ cts s$^{-1} \operatorname{arcsec}^{-1}$.

\section{Optical results}

In addition to the well known high-surface brightness inner disk, NGC 4569 clearly exhibits a low surface brightness outer disk in the $B$ and $R$ broad band images. The northern part reveals a distinct spiral arm, which connects with the higher surface brightness region. In addition to this arm a faint, structureless "disk" is visible which seems to be distorted roughly in the direction to IC 3583 . We also produced a $B-R$ color image (not displayed) indicating a bluer outer disk compared to the inner region. This may reflect the large dust content or higher metallicity of the inner high-surface brightness disk. In our continuum subtracted $\mathrm{H} \alpha+[\mathrm{N}$ II] image (Fig. 1 from Bomans et al. 2001) the extreme confinement of recent star formation to the inner part of the disk and its correspondence with the extension of X-ray emission (Sect. 4.1.1) is striking. 

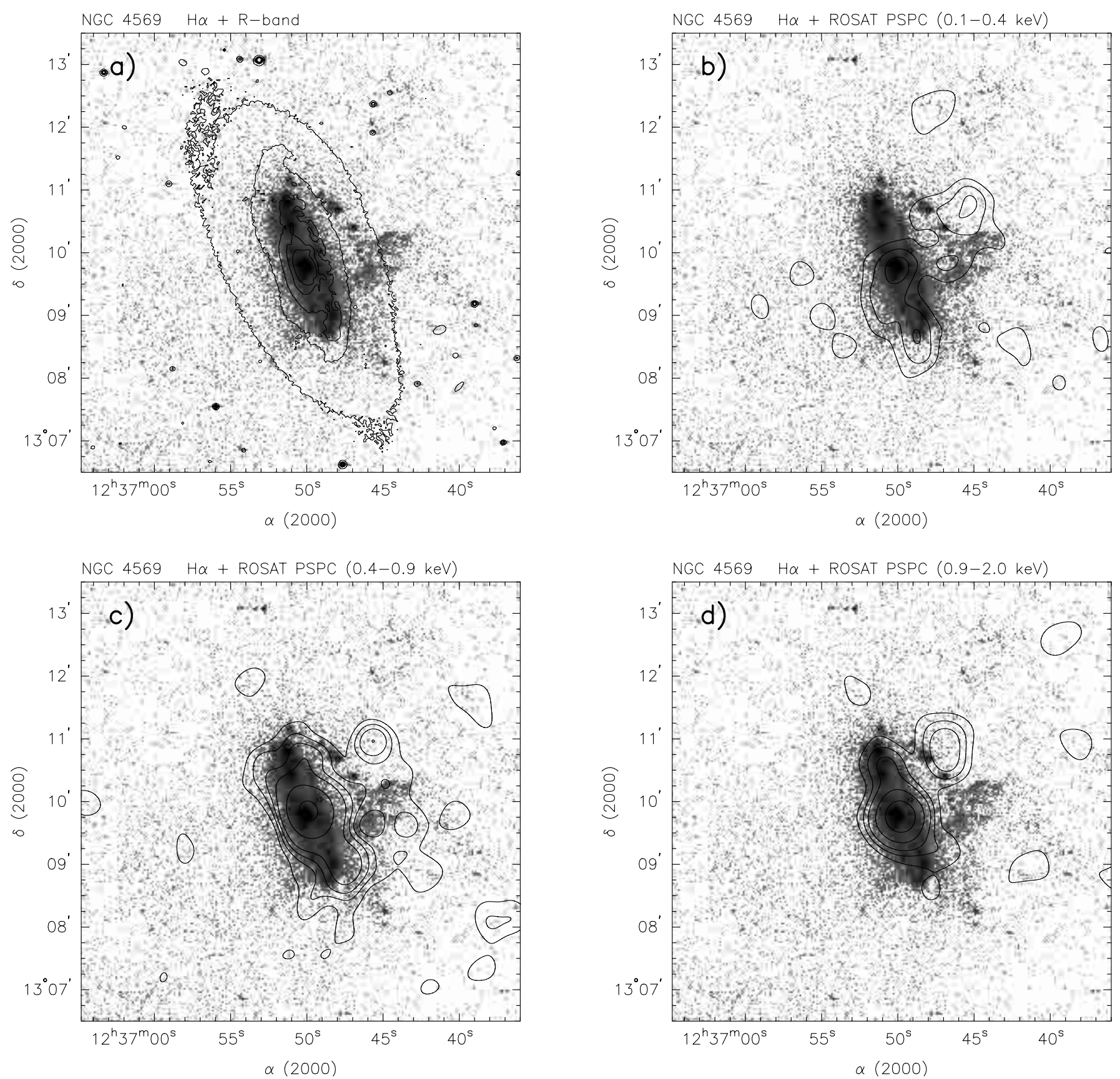

Fig. 4. a) $R$ band, b) $0.1-0.4 \mathrm{keV}$ X-ray, c) $0.5-0.9 \mathrm{keV}$ X-ray, and d) $0.9-2.0 \mathrm{keV}$ X-ray contours overlaid on an $\mathrm{H} \alpha+[\mathrm{N} \mathrm{II}]$ image of NGC 4569. The contour levels in the X-ray overlays b)-d) all start at $3 \sigma$ above the mean background. H $\alpha$ emission is confined within the inner $6 \mathrm{kpc}$ radius, similar to the Hi disk (compare Fig. 17b from Cayatte et al. 1990).

A specific attribute of NGC 4569 is the Hi deficiency and that the $21 \mathrm{~cm}$ emission is also confined to the central part (Cayatte et al. 1990). The Hi distribution has a ring-like structure and is located within the bright optical disk. Our optical images clearly reflect a high level of star formation activity concentrated to a relatively small area of the disk of NGC 4569 (about $12 \mathrm{kpc}$ in diameter). A dominant $\mathrm{H} \alpha$ source in the central region of NGC 4569 appears as unresolved giant HII region in our images. Faint diffuse emission is present between the large Hit regions in the high-surface brightness disk. The most remarkable structure in the $\mathrm{H} \alpha+[\mathrm{N} I \mathrm{II}$ image (Fig. 4) is the giant diffuse emission westward of the disk, forming a broad spur roughly pointing to the LINER nucleus of NGC 4569. This diffuse emission coincides with an extended X-ray component and may be due to ionized gas above the disk of NGC 4569, tracing a large-scale outflow or gaseous halo (see below). It is subject of a more comprehensive discussion in Bomans et al. (2001).

The optical images of IC 3583 reveal that it is a Magellanic-type irregular galaxy experiencing a phase of high star formation activity. The $B$ and $R$ band images 


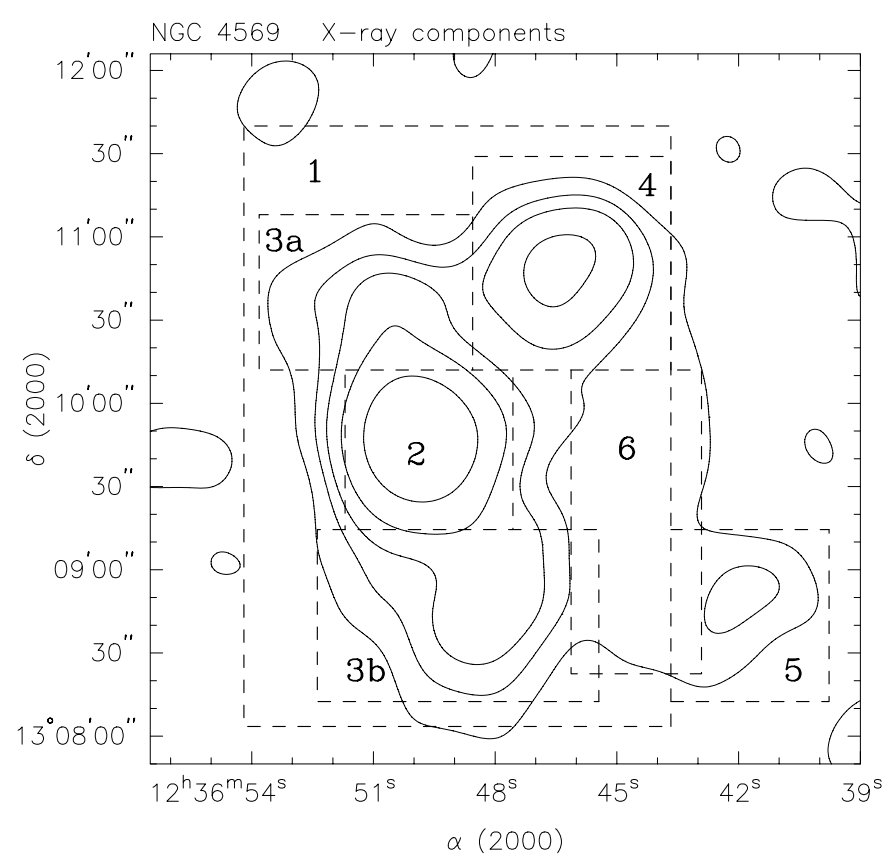

Fig. 5. Definition of disk, bulge, halo, and external source areas in the ROSAT PSPC image. Corresponding count rates and hardness ratios are listed in Table 5 .

exhibit a number of blue knots coinciding with large HII regions in our $\mathrm{H} \alpha+[\mathrm{N} \mathrm{II}]$ image. In addition to the bright clumps a faint, more extended component is present. The centroid of this faint emission is displaced with respect to the star formation regions. The overall color is very blue compared to NGC 4569, not unexpected for a dwarf irregular galaxy. A detailed structural analysis of IC 3583 is hampered by 3 bright stars near and on top of the galaxy body. Some diffuse HiI appears to be present between the HiI regions, but no large HiI shells as present in some other actively star-forming irregular galaxies (e.g. NGC 4449: Hunter \& Gallagher 1990; Bomans et al. 1997) could be detected. The most remarkable feature in IC 3583 is a narrow $\mathrm{H} \alpha$ spur at its southern $\operatorname{rim}$ pointing toward NGC 4569. Since it is also traceable in the $B$ image but not in the $R$ image, it represents probably a spur of star formation regions and not genuine diffuse HII.

\section{X-ray results}

\subsection{NGC 4569}

\subsubsection{Spatial analysis}

The X-ray emission in the direction of NGC 4569 is clearly extended. From the superposition of the PSPC contours onto the optical $B$ band image of NGC 4569 (Fig. 3a) one can distinguish different components in X-rays. In the following the numbers refer to the division of the X-ray area into different zones and the compilation of the X-ray parameters (Table 5, see also Fig. 5). The maximum of the X-ray emission originates from the nuclear region of the galaxy (area 2), but disk emission is also clearly visible (area 3a and 3b). Additionally, the X-ray disk component fits well with the extension of the Hi contours
Table 5. PSPC count rates and hardness ratios of the X-ray components of NGC 4569, as labeled in Fig. 5.

\begin{tabular}{lll}
\hline Component & $\begin{array}{l}\text { Count rates }^{a} \\
{\left[10^{-3} \text { cts s}^{-1}\right]}\end{array}$ & Hardness ratio $^{b}$ \\
\hline 1 & $33.8 \pm 1.9$ & $0.51 \pm 0.04$ \\
$1^{c}$ & $27.9 \pm 1.4$ & $0.56 \pm 0.04$ \\
2 & $14.4 \pm 0.9$ & $0.77 \pm 0.05$ \\
$3 \mathrm{a}$ & $3.4 \pm 0.6$ & $0.99 \pm 0.12$ \\
$3 \mathrm{~b}$ & $5.3 \pm 0.7$ & $0.06 \pm 0.10$ \\
4 & $5.8 \pm 0.7$ & $0.26 \pm 0.09$ \\
5 & $1.9 \pm 0.3$ & $0.19 \pm 0.15$ \\
6 & $2.7 \pm 0.4$ & $0.13 \pm 0.13$ \\
\hline
\end{tabular}

${ }^{a}$ Background subtracted.

${ }^{b}$ For definition see text.

${ }^{c}$ Excluding area 4 (see text).

(Cayatte et al. 1990) and the $\mathrm{H} \alpha+[\mathrm{N}$ II] emission, whereas the optical disk is much widerly extended (Fig. 4a). Two nearby X-ray components are probably not directly connected with NGC 4569: The X-ray source $2^{\prime}$ northwest of the center (area 4) may be produced in the stellar disk of NGC 4569, but it is more likely associated with an independent foreground/background source not related to the galaxy. However, up to now no optical candidate has been detected for this source. A further source southwest of the center of NGC 4569 (area 5) can be identified as the background galaxy VPC 1071 visible in our $B$ and $R$ band images. Diffuse X-ray emission most likely in connection with NGC 4569 is found west of the center (area 6), its soft spectral distribution lets us suggest a hot gas bubble as the source of diffuse emission and indicates its origin from above the disk (see below).

Table 5 compiles count rates and hardness ratios of the different X-ray emission components in the direction of NGC 4569 in comparison to the total area 1. We define the hardness ratio by $H R=\frac{B-A}{B+A}$ with $A$ and $B$ being the counts summed over the 11-41 and 52-201 energy channels, respectively (with a conversion factor $E[\mathrm{keV}]=$ channel number $/ 100)^{1}$. It is quite obvious that the nuclear emission of NGC 4569 is strongly absorbed (hardness ratio $H R=0.8$ ), whereas the contribution from area 6 is much softer. This diffuse emission is of particular interest for the possible existence of an extraplanar X-ray component in NGC 4569 and will be discussed in detail in Sect. 5.3. Another interesting point is the significant difference in hardness ratio between the northern and southern disk part. This is corroborated by the comparison of the X-ray emission in different PSPC spectral ranges (Fig. 4): there is no soft emission $(0.1-0.4 \mathrm{keV})$ visible from the northern disk, whereas it is pronounced in the southern disk.

Although the exposure time of the HRI observation was larger by a factor 1.3 than the one of the PSPC observation, only the nuclear source can be clearly

1 The definition of $H R$ here is slightly different to the one used by Junkes et al. (1995). 

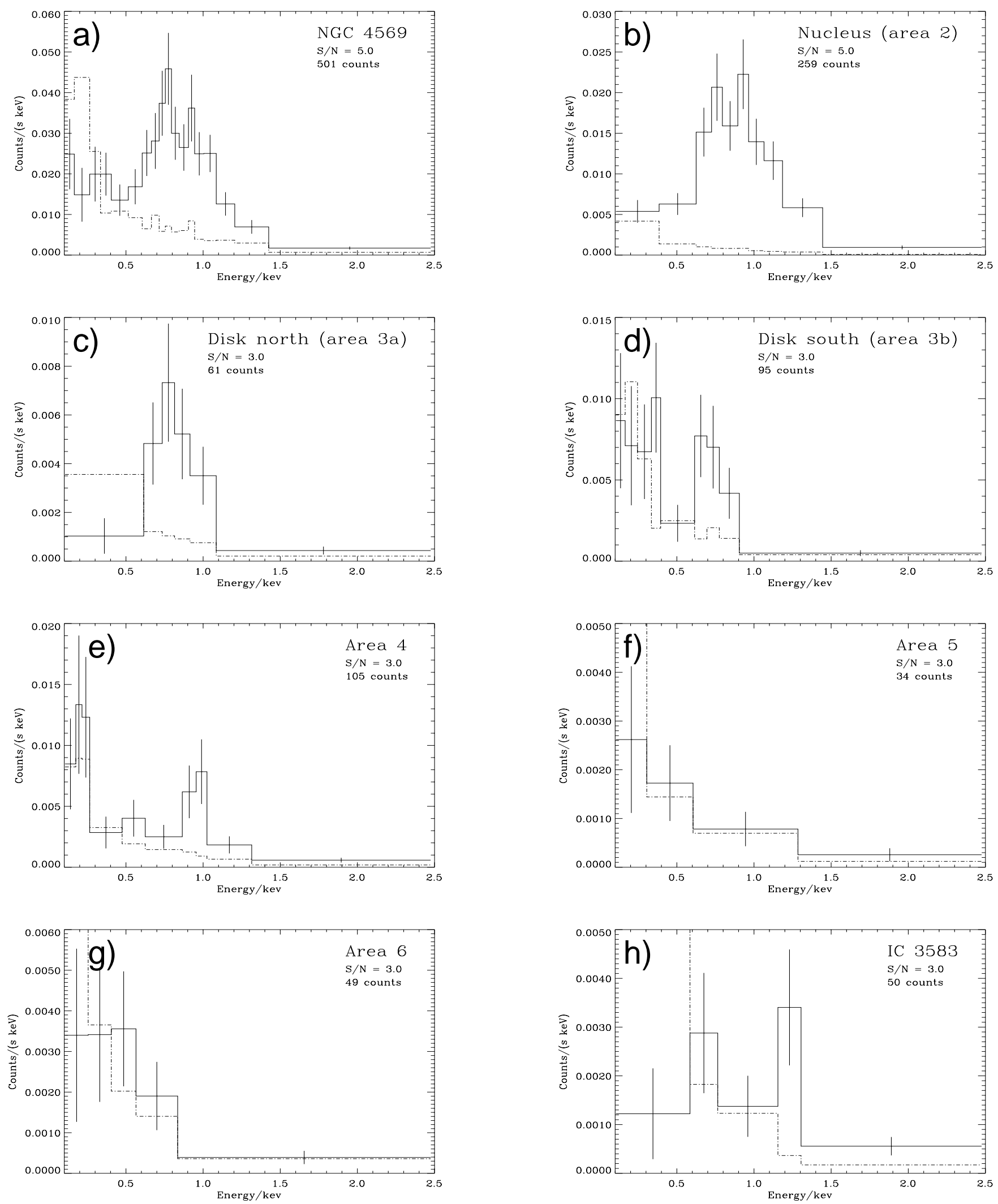

Fig. 6. Background-subtracted ROSAT PSPC spectra of a) NGC 4569 (area 1 excluding area 4), b) the nucleus (area 2), c) the northern disk (area 3a), d) the southern disk (area 3b), e) the northwestern point source (area 4), f) the southwestern source (area 5), g) the western diffuse component (area 6), and $\mathbf{h}$ ) IC 3583 in the energy range of $0.1-2.4 \mathrm{keV}$. The photons of each spectrum were binned to get a signal-to-noise ratio of 5 for the entire galaxy and the nucleus and 3 for the other sources. In each case the dashed-dotted line represents the contribution of the background. 
identified, but is unresolved at length scales of $5^{\prime \prime}$ or 400 pc (Fig. 3b). Considering the HRI detector to be roughly three times less sensitive than the PSPC, the count rate of the HRI observations from the nucleus with $4.6 \times 10^{-3} \mathrm{cts} \mathrm{s}^{-1}$ (Table 4 ) is consistent with the one of the PSPC $\left(14.4 \times 10^{-3}\right.$ cts s $^{-1}$; Table 5$)$. The extended disk emission from NGC 4569, observed with the PSPC, would lie less than $1 \sigma$ above the HRI detector background, which is at $3.7 \times 10^{-3} \operatorname{cts~s}^{-1} \operatorname{arcsec}^{-2}$, and is therefore not detectable in the HRI image (Fig. 3b).

\subsubsection{The $X$-ray spectrum}

In Fig. 6 the PSPC spectra of the different components of NGC 4569 (labeled in Fig. 5) and from IC 3583 are given. Whereas the distribution from the entire galaxy contains a significant portion in the lower energy channels below $0.5 \mathrm{keV}$, which is assumed to be mainly attributed to emission from the southern disk and halo of NGC 4569, the soft spectral part of the nuclear region is significantly absorbed. The corresponding hardness ratios also support this picture $(H R=0.77 \pm 0.05$ for area 2$)$ and an overall softer disk and halo component $(H R=0.56 \pm 0.04$ for area 1 , excluding area 4$)$. The much softer spectrum of the peak emission in area $4(H R=0.26 \pm 0.09)$ points to a foreground location of this source.

As already mentioned area $3 \mathrm{a}$ has a much harder spectral distribution compared to area $3 \mathrm{~b}$ (Table 5). Additionally, the $0.1-2.4 \mathrm{keV} \mathrm{X}$-ray emission from the disk seems to be slightly asymmetric toward the south. We will discuss possible explanations for the difference in the disk components in Sects. 5.2 and 5.5. The very soft component west of the disk (area 6) is of particular interest and will be considered in Sect. 5.3.

The raw background-subtracted X-ray spectra from the entire galaxy excluding area 4 and from the nucleus are composed by $501 \pm 25$ and $259 \pm 16$ counts, respectively. They are fitted with several single component models, like a Raymond-Smith model of a hot thermal plasma (Raymond \& Smith 1977: RS), a power-law model (PO), and a thermal Bremsstrahlung model (BS). Additionally, we fit the spectrum of the galaxy by a two-component ( $\mathrm{RS}+\mathrm{PO})$ model and by a single $\mathrm{RS}$ component of two different fixed abundances (i.e. $0.1 Z_{\odot}$ and $1 Z_{\odot}$ ). The resulting parameter combinations are listed in Tables 6 and 7 .

For all fits to the spectra of nucleus and total galaxy, except the RS model with solar metallicity for the nucleus, the corresponding absorption values reach at least the Galactic Hi column density of $2.47 \times 10^{20} \mathrm{~cm}^{-2}$ in the direction of NGC 4569 (Dickey \& Lockman 1990). From the matching spatial distributions in X-rays and HI an additional inherent absorption in NGC 4569 is expected. HI maps of NGC 4569 (Cayatte et al. 1990) give column densities above $5 \times 10^{20} \mathrm{~cm}^{-2}$ (up to $2 \times 10^{21} \mathrm{~cm}^{-2}$ in the northern part of the disk component). This, however, would preferentially support the PO model for the nucleus, since this is the only one with an absorption component higher than $10^{21} \mathrm{~cm}^{-2}$.

The best spectrum fit of the whole NGC 4569 is achieved with a combined $\mathrm{RS}+\mathrm{PO}$ model for the X-ray emission in the ROSAT band, accounting for contributions from the hot gas in the disk and the halo and from possible central sources, like an AGN, high-mass X-ray binaries (HMXBs), and SNRs in the starburst region and the disk. Since resolution and intensity of the PSPC spectrum are not sufficient to determine the element abundances in the Raymond-Smith plasma directly by fitting, only fixed values are applied to find an appropriate fit. We test models with $0.1,0.3,0.6$, and 1 times solar abundances. The best fit for the nuclear component results in a pure RS model with low metallicity of $0.1 Z_{\odot}$. Increasing the metallicity results in a continuous decrease of both $N_{\mathrm{H}}$ and the fit quality. It has no influence on the plasma temperature. These spectral fits and the mismatch of a single PO model support the conclusion that the central X-ray emission is not dominated by an active non-thermal nuclear source, which agrees with the conclusion result drawn from the absence of hard X-rays in ASCA observations.

\subsection{The nearby irregular dwarf galaxy IC 3583}

IC 3583 has been tentatively identified in the Einstein observation of NGC 4569 (Fabbiano et al. 1992), however, with a significant position offset. In our ROSAT image of the field, IC 3583 appears as a compact source. In Fig. 7 the contours of the X-ray emission in the energy range $0.1-2.4 \mathrm{keV}$ are overlaid onto the $B$ band and $\mathrm{H} \alpha+[\mathrm{NII}]$ image. The compact X-ray source at the position of IC 3583 has been detected by the PSPC with a count rate of $(2.8 \pm 0.4) \times 10^{-3} \mathrm{cts} \mathrm{s}^{-1}$ and by the HRI with $(5.9 \pm 1.9) \times 10^{-4}$ cts s$^{-1}$, respectively. It is not possible, however, to spatially distinguish emission from IC 3583 from a foreground star lying exactly on the line of sight to the galaxy. For this reason we also investigate its spectrum. After correction for the background we have a total of $50 \pm 8$ counts in the $18 \mathrm{ksec}$ exposure. The resulting hardness ratio is $0.95 \pm 0.12$ which indicates a significant foreground+intrinsic absorption and/or a rather hard X-ray spectrum. This supports the identity of the X-ray source with the irregular galaxy IC 3583 and not to be a foreground star. A $3 \sigma$ spectrum (i.e. a signal-to-noise ratio of 3 in each bin) results in only 5 bins (Fig. $6 \mathrm{~h}$ ). Fits of standard models (e.g. power law and Raymond-Smith thermal plasma fit) to such a spectrum are generally invalid and not suitable for quantitative information.

\section{Discussion}

\subsection{Nuclear activity in NGC 4569}

The X-ray emission from the nucleus of NGC 4569 is spatially not resolved by ROSAT, so that an upper limit of $\sim 400$ pc can be derived for its total extent from the HRI. From high-resolution optical and UV data the nature of 
Table 6. Fit results on the X-ray spectrum from NGC 4569 (area 1 excluding area 4).

\begin{tabular}{|c|c|c|c|c|c|c|c|c|c|}
\hline $\begin{array}{l}\text { Model } \\
\text { (1) }\end{array}$ & $\begin{array}{l}N_{\mathrm{H}} \\
(2)\end{array}$ & $\begin{array}{l}k T \\
(3)\end{array}$ & $\begin{array}{l}\Gamma \\
(4)\end{array}$ & $\begin{array}{l}Z \\
(5)\end{array}$ & $\begin{array}{l}\text { Norm } \\
(6)\end{array}$ & $\begin{array}{l}\text { Red. } \chi^{2} \\
(7)\end{array}$ & $\begin{array}{l}\text { d.o.f. } \\
(8)\end{array}$ & $\begin{array}{l}F_{\mathrm{X}} \\
(9)\end{array}$ & $\begin{array}{l}L_{\mathrm{X}} \\
(10)\end{array}$ \\
\hline $\mathrm{RS}$ & $2.39_{-0.57}^{+0.83}$ & $0.63_{-0.14}^{+0.12}$ & & 0.1 & 42 & 1.2 & 15 & $0.37_{-0.03}^{+0.01}$ & $1.25_{-0.10}^{+0.03}$ \\
\hline $\mathrm{RS}$ & $0.20_{-0.01}^{+0.39}$ & $0.63_{-0.18}^{+0.16}$ & & 1 & 5.8 & 2.4 & 15 & $0.22_{-0.03}^{+0.01}$ & $0.74_{-0.10}^{+0.03}$ \\
\hline BS & $5.79_{-0.75}^{+1.06}$ & $0.45_{-0.06}^{+0.05}$ & & & 2.1 & 1.2 & 15 & $0.91_{-0.10}^{+0.06}$ & $3.06_{-0.33}^{+0.21}$ \\
\hline $\mathrm{PO}$ & $9.39_{-0.86}^{+1.33}$ & & $3.6 \pm 0.2$ & & 13.1 & 1.5 & 15 & $5.21_{-1.47}^{+2.17}$ & $17.6_{-5.0}^{+7.4}$ \\
\hline $\mathrm{RS}+\mathrm{PO}$ & $4.91_{-1.13}^{+3.21}$ & $0.44_{-0.12}^{+0.19}$ & $2.7_{-2.0}^{+1.3}$ & 0.1 & $\begin{array}{l}5.3(\mathrm{PO}) \\
34(\mathrm{RS})\end{array}$ & 0.9 & 13 & $\begin{array}{l}0.84_{-0.42}^{+3.72} \\
(0.26 \pm 0.04)\end{array}$ & $\begin{array}{l}2.84_{-1.42}^{+12.6} \\
(0.88 \pm 0.13)\end{array}$ \\
\hline $\mathrm{RS}+\mathrm{PO}$ & $5.85_{-0.71}^{+0.79}$ & $0.46_{-0.11}^{+0.18}$ & $3.0 \pm 0.4$ & 1 & $\begin{array}{l}7.4(\mathrm{PO}) \\
3.3(\mathrm{RS})\end{array}$ & 0.9 & 13 & $\begin{array}{l}1.31_{-0.54}^{+0.92} \\
\left(0.12_{-0.02}^{+0.01}\right)\end{array}$ & $\begin{array}{l}4.42_{-1.82}^{+3.10} \\
\left(0.41_{-0.06}^{+0.03}\right)\end{array}$ \\
\hline
\end{tabular}

Column (1): spectral models: BS = thermal Bremsstrahlung, RS = Raymond-Smith, $\mathrm{PO}=$ power law;

Column (2): column density in units of $10^{20} \mathrm{~cm}^{-2}$;

Column (3): plasma temperature in units of $\mathrm{keV}$;

Column (4): photon index;

Column (5): metallicity in units of $Z_{\odot}$ (fixed);

Column (6): scaling factor: for BS in units of $\left(10^{-18} /\left(4 \pi D^{2}\right)\right) \int n_{\mathrm{e}} n_{\mathrm{I}} \mathrm{d} V, n_{\mathrm{e}}, n_{\mathrm{I}}=$ electron and ion densities $\left(\mathrm{cm}^{-3}\right)$; for RS in units of $\left(10^{-19} /\left(4 \pi D^{2}\right)\right) \int n_{\mathrm{e}}^{2} \mathrm{~d} V, n_{\mathrm{e}}=$ electron density $\left(\mathrm{cm}^{-3}\right)$; for PO in units of $10^{-5}$ photons keV $\mathrm{km}^{-1} \mathrm{~s}^{-1}$ at $1 \mathrm{keV}$;

Column (7): reduced $\chi^{2}$;

Column (8): degrees of freedom;

Column (9): unabsorbed X-ray flux in units of $10^{-12} \mathrm{erg}^{-2} \mathrm{~s}^{-1}$. Values in brackets give the contribution of the thermal component;

Column (10): X-ray luminosity in units of $10^{40} \mathrm{erg} \mathrm{s}^{-1}$. Values in brackets give the contribution of the thermal component.

Table 7. Fit results on the X-ray spectrum from the nucleus of NGC 4569 (area 2).

\begin{tabular}{llllllllll}
\hline $\begin{array}{l}\text { Model } \\
(1)\end{array}$ & $\begin{array}{l}N_{\mathrm{H}} \\
(2)\end{array}$ & $k T$ & $\Gamma$ & $\begin{array}{l}Z \\
(3)\end{array}$ & $\begin{array}{l}\text { Norm } \\
(4)\end{array}$ & $\begin{array}{l}\text { Red. } \chi^{2} \\
(7)\end{array}$ & $\begin{array}{l}\text { d.o.f. } \\
(8)\end{array}$ & $\begin{array}{l}F_{\mathrm{X}} \\
(9)\end{array}$ & $\begin{array}{l}L_{\mathrm{X}} \\
(10)\end{array}$ \\
\hline & & & & & & & & & \\
$\mathrm{RS}$ & $4.10_{-0.91}^{+1.54}$ & $0.77_{-0.13}^{+0.11}$ & & 0.1 & 27 & 0.5 & 7 & $0.24 \pm 0.01$ & $0.81 \pm 0.03$ \\
$\mathrm{RS}$ & $1.21_{-0.37}^{+0.53}$ & $0.84_{-0.05}^{+0.04}$ & & 1 & 4.9 & 1.7 & 7 & $0.15_{-0.01}^{+0.02}$ & $0.52_{-0.04}^{+0.05}$ \\
$\mathrm{BS}$ & $7.41_{-1.29}^{+2.09}$ & $0.56_{-0.08}^{+0.10}$ & & & 9.4 & 1.5 & 7 & $0.46_{-0.04}^{+0.05}$ & $1.56_{-0.15}^{+0.17}$ \\
$\mathrm{PO}$ & $10.5 \pm 2.0$ & & $3.2_{-0.4}^{+0.3}$ & & 8.6 & 2.4 & 7 & $1.8_{-0.8}^{+1.1}$ & $6.0_{-2.6}^{+3.8}$ \\
\hline
\end{tabular}

Column (1): spectral models: BS = thermal Bremsstrahlung, RS = Raymond-Smith, $\mathrm{PO}=$ power law;

Column (2): column density in units of $10^{20} \mathrm{~cm}^{-2}$;

Column (3): plasma temperature in units of $\mathrm{keV}$;

Column (4): photon index;

Column (5): metallicity in units of $Z_{\odot}$;

Column (6): scaling factor: for BS in units of $\left(10^{-19} /\left(4 \pi D^{2}\right)\right) \int n_{\mathrm{e}} n_{\mathrm{I}} \mathrm{d} V, n_{\mathrm{e}}, n_{\mathrm{I}}=$ electron and ion densities $\left(\mathrm{cm}^{-3}\right)$; for RS in units of $\left(10^{-19} /\left(4 \pi D^{2}\right)\right) \int n_{\mathrm{e}}^{2} \mathrm{~d} V, n_{\mathrm{e}}=$ electron density $\left(\mathrm{cm}^{-3}\right)$; for PO in units of $10^{-5}$ photons keV $\mathrm{cm}^{-2} \mathrm{~s}^{-1}$ at $1 \mathrm{keV}^{-}$

Column (7): reduced $\chi^{2}$;

Column (8): degrees of freedom;

Column (9): unabsorbed X-ray flux in units of $10^{-12} \mathrm{erg}^{-2} \mathrm{~s}^{-1}$;

Column (10): X-ray luminosity in units of $10^{40} \mathrm{erg} \mathrm{s}^{-1}$.

the core of NGC 4569 is equivocal: it consists of a normal bulge population, a supergiant dominated A-type population within a radius of $20 \mathrm{pc}$, and a very compact, UVluminous core with a possible slight extension in UV and in the $V$ band (Barth et al. 1998; Barth \& Shields 2000). Keel (1996) studied two possibilities for the core: (a) an active accretion-powered nucleus and (b) a compact and very luminous central star cluster, as large as the core of 30 Dor but more than 6 mag brighter. He concluded that a possible young starburst would be required as an explanation for the existence of this unusually high number of A supergiants, while the spectral shape, flat in the UV with a steep drop in the optical, is not expected for an AGN.

From the X-ray data two interpretations for the nuclear emission seem possible: X-ray emission from an 

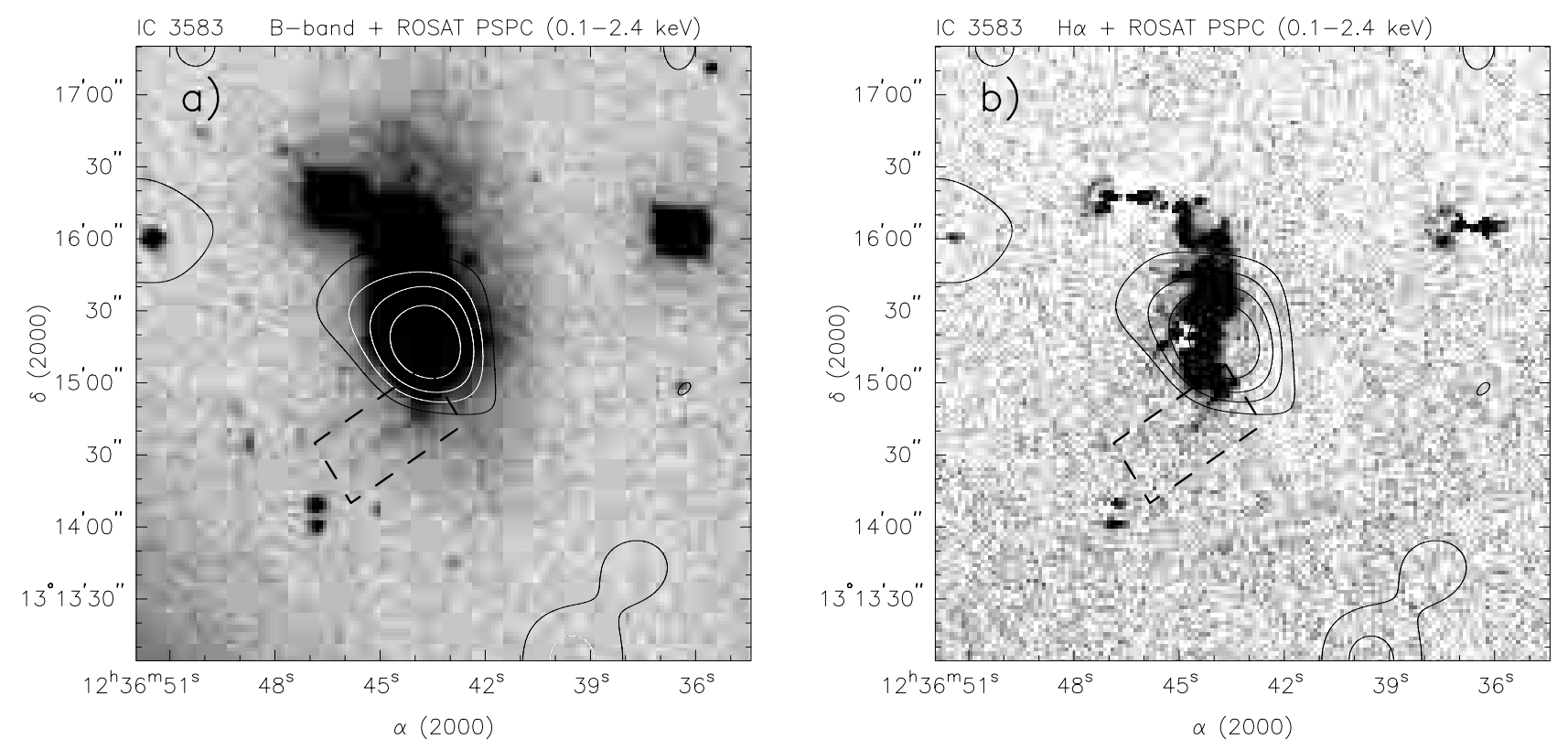

Fig. 7. ROSAT PSPC contours in a $4^{\prime} \times 4^{\prime}$ around IC 3583 overlaid onto the a) $B$ band and b) $\mathrm{H} \alpha+[\mathrm{N}$ II] image. The X-ray contours are 3, 5, 7, and $10 \sigma$ above the mean background of $5.3 \times 10^{-7} \mathrm{cts} \mathrm{s}^{-1} \operatorname{arcsec}^{-1}\left(\sigma=1 \times 10^{-7} \mathrm{cts} \mathrm{s}^{-1} \mathrm{arcsec}^{-1}\right)$. A narrow $\mathrm{H} \alpha$ spur is visible at the southern end of IC 3583, also weakly traceable in the $B$ band, pointing toward NGC 4569. The spur is marked by the dashed box in each image.

unresolved compact nuclear source is associated either with the LINER-type nucleus of NGC 4569 in the optical, or with a slightly extended nuclear starburst region, similar to the ones found in NGC 1808 (Junkes et al. 1995), NGC 2903 (Tschöke et al. 2001), and NGC 4303 (Colina \& Arribas 1999; Tschöke et al. 2000) at a scale of $\sim 1 \mathrm{kpc}$.

Since Terashima et al. (2000) do not find any compact nuclear source in the $2-7 \mathrm{keV}$ band of their ASCA data, they exclude an AGN in NGC 4569, unless the obscuring column density is with $N_{\mathrm{H}}>10^{24} \mathrm{~cm}^{-2}$ implausibly high. This conclusion is supported by our findings that the spectrum of the nuclear source in NGC 4569 can well be fitted with a RS spectrum, either contributed by cumulative stellar winds in a central super star cluster (SSC), expanding supernovae of type II (SNeII) bubbles from extinguished massive stars in these SSCs, or a combination of both. Nonetheless, with the spectral resolution of the ROSAT PSPC because of the low photon statistics of the central source (10 bins for a $5 \sigma$ spectrum) it would not be possible to clearly determine the nature of the core emission, whether of thermal or non-thermal origin. Moreover, the column density of the RS model with metallicity $Z=0.1 Z_{\odot}$ lies below the Galactic value and even the model with solar metallicity below the observed intrinsic value for the nuclear region. On the other hand, the extended hard X-ray emission is consistent with a collection of discrete hard sources, like X-ray binaries.

The total X-ray luminosity of $1-4 \times 10^{40} \mathrm{erg} \mathrm{s}^{-1}$ (depending on the applied model) is not too high to be explained solely by strong star-forming activity in NGC 4569. From our analysis, we can interpret the spectrum of the total X-ray emission from NGC 4569 in two possible ways: at first, the soft X-ray emission contains contributions from two components - a hot thermal plasma (RS) from the starburst as well as a PO component which can either be attributed to an unresolved AGN component or more likely to HMXBs and SNRs in a compact nuclear starburst. Secondly, the central X-ray source is dominated by a very compact luminous central SSC, described by a RS plasma model in the ROSAT energy band.

Further evidence for the absence of an AGN in NGC 4569 arises from the X-ray to $\mathrm{H} \alpha$ luminosity ratio. For AGNs this ratio is up to 100 times higher than for pure nuclear starbursts. With an $\mathrm{H} \alpha$ flux of $9.4 \times 10^{40} \mathrm{erg} \mathrm{s}^{-1}$ for NGC 4569 (Kennicutt et al. 1994) we derive $\log \left(L_{\mathrm{X}} / L_{\mathrm{H} \alpha}\right)=-1.1$ to -0.3 , consistent with other starburst galaxies, like e.g. NGC 1069 or NGC 1097 (Pérez-Olea \& Colina 1996), while typical AGNs have values larger than -0.2 .

\subsection{Disk emission}

The total disk emission in X-rays from either side (NE vs. SW) is comparable, their distributions throughout the ROSAT band, however, differ significantly. The contribution from the northern part (area 3a) is clearly harder (Table 5). This may be caused by a higher fraction of hard point sources, like HMXBs and SNRs, and less contribution from soft diffuse X-ray emission compared to the southern region. The almost equal HI-column densities derived by Cayatte et al. (1990) do not allow to make any intrinsic absorption responsible. Another possibility takes the interaction with the Virgo ICM into account; we propose that the southern soft component is a result from the central starburst, as supposed for the western soft X-rays 
(Sect. 5.3), and experiences a strong impact from the surrounding hot ICM. This interpretation will be discussed in context with the entire galaxy in Sect. 5.5. Nevertheless, the difference in X-ray emission between the northern and southern disk component is not yet fully understood.

\subsection{Contribution from the halo}

NGC 4569 has an inclination of $i \approx 64^{\circ}$. The galaxy shows extended soft X-ray emission with contributions from outside the nucleus and outside the disk. In contrast to edgeon galaxies, where emission components from disk and halo can be quite easily distinguished (e.g. Vogler et al. 1996), it is more difficult to disentangle these components in the case of NGC 4569. Additional difficulties arise from the nearby two foreground/background sources northwest and southwest of the nucleus.

The overall distribution of the soft X-ray emission appears to be very asymmetric with a spur of soft emission extending from the galaxy center and some additional diffuse patches both west of the galaxy. We mark a rectangle area of $50^{\prime \prime} \times 100^{\prime \prime}$ (Fig. 5, area 6) taking care to avoid any contamination by the source in area 4 . The total number of counts in the area 6 with boundaries as defined in Fig. 5 is $49 \pm 7$ after background subtraction. If we apply a RS model with strongly constraint parameters, i.e. assuming a reasonable column density value of $N_{\mathrm{H}}=3 \times 10^{20} \mathrm{~cm}^{-2}$ (Galactic foreground value; Hi observations from Cayatte et al. (1990) give $N_{\mathrm{H}}$ values below $5 \times 10^{20} \mathrm{~cm}^{-2}$ in the relevant halo region) and solar abundances, we obtain a plasma temperature of about $0.2 \mathrm{keV}$, resulting in a flux of $2.4 \times 10^{-14} \mathrm{erg} \mathrm{s}^{-1} \mathrm{~cm}^{-2}$ in the ROSAT band and an $\mathrm{X}$-ray luminosity of $8 \times 10^{38} \mathrm{erg} \mathrm{s}^{-1}$. Even abundances of $0.1 Z_{\odot}$ do not alter the result significantly. If we take $H R=0.39$ as an upper limit, a corresponding RS spectrum with $N_{\mathrm{H}}=3 \times 10^{20} \mathrm{~cm}^{-2}$ would yield a temperature of $0.24 \mathrm{keV}$. A column density of $5 \times 10^{20} \mathrm{~cm}^{-2}$ would drop the temperature to $0.19 \mathrm{keV}$. With a lower $H R$ of 0.13 and $N_{\mathrm{H}}=3 \times 10^{20} \mathrm{~cm}^{-2}$ we again get a plasma temperature of $0.2 \mathrm{keV}$. Again, an increase of the absorption component will decrease the corresponding temperature for the observed spectral distribution. Hence, for the X-ray emission from area 6 we deduce a low plasma temperature of approximately $0.2 \mathrm{keV}$, similar to NGC 4449 (Bomans et al. 1997) or NGC 1705 (Hensler et al. 1998).

The spatial coincidence of the western soft X-ray emission with an extended $\mathrm{H} \alpha$ structure supports the interpretation as hot halo gas being ejected from the central starburst region strikingly Gas expanding from the disk is also visible in the radial velocities of this $\mathrm{H} \alpha$ emission (Bomans et al. 2001; Boselli, priv. comm.). Similar correlations were observed in a number of nearby starburst galaxies (e.g. M 82: Strickland et al. 1997; NGC 253: Strickland et al. 2000; Shopbell \& Bland-Hawthorn 1998; Lehnert et al. 1999; NGC 3628: Dahlem et al. 1996; NGC 4666: Dahlem et al. 1997) and are interpreted as a signature for hot gas outflow from the starburst center.

\subsection{Physical properties of the hot halo}

In general, two different morphologies for a hot gaseous halo are possible, depending on the types of outflow mechanisms from the galactic disk: (a) If the star formation takes place in a compact central starburst, a conical outflow of gas occurs, driven by stellar winds of massive stars and SNII explosions. A similar morphology arises from an AGN producing a jet. (b) If the star formation is distributed over large parts of the galactic disk, several distinct star-forming regions blow out hot gas into a more extended region around the disk. Another possibility is a mixture of these two morphological cases.

If we assume a simplified geometry of an outflow from a central starburst for the diffuse X-ray gas in area 6 (a cylindrical shape with a height of $6 \mathrm{kpc}$ and a diameter of $4 \mathrm{kpc}$, and therefore a volume $V$ of $8 \times 10^{10} \mathrm{pc}^{3}$ ), we can estimate the mean electron density of the gas. Applying solar abundances, the derived plasma temperature of $0.2 \mathrm{keV}$ is consistent with an emissivity $\Lambda_{\mathrm{R}}(T)$ of $2.5 \times 10^{-23} \mathrm{erg} \mathrm{cm} \mathrm{cm}^{3} \mathrm{~s}^{-1}$ in the energy range of $0.1-$ $2.4 \mathrm{keV}$. This value is most sensitive to the chosen metallicity; for $0.3 Z_{\odot}$ it is about $1 \times 10^{-23} \mathrm{erg} \mathrm{cm}^{3} \mathrm{~s}^{-1}$. With $L_{\mathrm{X}}=n_{\mathrm{e}}^{2} \Lambda_{\mathrm{R}}(T) V$ we derive a mean electron density of $n_{\mathrm{e}} \approx 4 \times 10^{-3} f^{-1 / 2} \mathrm{~cm}^{-3}$, with $f$ being the volume filling factor of the halo gas. The total mass of the hot gas is then $M \approx 7 \times 10^{6} f^{1 / 2} M_{\odot}$, and the total thermal energy is $E_{\mathrm{th}} \approx 4 \times 10^{54} f^{1 / 2} \mathrm{erg}$. Pressure $P=2 n k T$ and cooling time $\tau_{\text {cool }}=3 k T / n \Lambda$ amount to $3 \times 10^{-12} f^{-1 / 2} \mathrm{dyn} \mathrm{cm}^{-2}$ and $3 \times 10^{8} f^{1 / 2} \mathrm{yr}$, respectively.

A spherical X-ray halo would also be realistic, since the western X-ray halo is clearly extended toward the south, while its extension and shape to the north cannot be determined because of the X-ray source in the area 4 . In order to derive the halo properties any contamination of the disk emission (components 2 and $3 \mathrm{~b}$ ) must be avoided. Therefore, we apply the shape of a spherical X-ray halo around NGC 4569 with a radius of $9 \mathrm{kpc}$ of which the western part 6 (Fig. 5) represents only the upper half as a $4.5 \mathrm{kpc}$ high segment starting already at a height of $4.5 \mathrm{kpc}$ above the disk plane. Its volume then amounts to $2 \times 10^{11} \mathrm{pc}^{3}$. An X-ray luminosity of $L_{\mathrm{X}} \approx 1.5 \times 10^{39} \mathrm{erg} \mathrm{s}^{-1}$ for this part only yields an electron density of $3 \times 10^{-3} f^{-1 / 2} \mathrm{~cm}^{-3}$, a thermal energy of $6 \times 10^{55} f^{1 / 2} \mathrm{erg}$, and a cooling timescale of $3 \times 10^{8} f^{1 / 2} \mathrm{yr}$. The total halo mass then amounts to $1.5 \times 10^{7} f^{1 / 2} M_{\odot}$.

A compilation of the physical properties of the halo of NGC 4569 and a few other observed X-ray halos are given for comparison in Table 8. The X-ray halo of NGC 4569 is in extension, luminosity, mass, and density quite similar to the one of NGC 253. Both galaxies reveal a few times lower X-ray luminosity, at most $10^{39} \mathrm{erg} \mathrm{s}^{-1}$, while other $\mathrm{X}$-ray halos have luminosities of several times $10^{39} \mathrm{erg} \mathrm{s}^{-1}$. The electron densities of NGC 4569 and NGC 253 are also somewhat lower compared to the other galaxies. The two irregular galaxies M 82 and NGC 4449 may not directly be comparable to the rest of the sample because of their different morphology, metallicity, and mass. 
Table 8. Properties of a number of observed X-ray halos.

\begin{tabular}{|c|c|c|c|c|c|c|c|c|}
\hline & $\begin{array}{l}\text { Type } \\
(1)\end{array}$ & $\begin{array}{l}k T \\
(2)\end{array}$ & $\begin{array}{l}L_{\mathrm{X}} \\
(3)\end{array}$ & $\begin{array}{l}n_{\mathrm{e}} \\
(4)\end{array}$ & $\begin{array}{l}M \\
(5)\end{array}$ & $\begin{array}{l}\tau_{\text {cool }} \\
(6)\end{array}$ & $\begin{array}{l}h \\
(7)\end{array}$ & $\begin{array}{l}\text { Ref. } \\
(8)\end{array}$ \\
\hline M 82 & pec & $0.4-0.6$ & $1.9 \times 10^{40}$ & 0.02 & $1.3 \times 10^{8}$ & $1 \times 10^{8}$ & 6 & 1 \\
\hline NGC 4449 & $\mathrm{Im}$ & 0.2 & $7 \times 10^{38}$ & 0.03 & $2 \times 10^{6}$ & $2 \times 10^{7}$ & 1.8 & 2 \\
\hline NGC 253 & $\mathrm{Sc}$ & 0.2 & $1.6 \times 10^{39}$ & $2 \times 10^{-3}$ & $5 \times 10^{6}$ & $2 \times 10^{8}$ & 10 & 3,4 \\
\hline NGC 3079 & $\mathrm{Sm}$ & 0.3 & $5.8 \times 10^{39}$ & $7 \times 10^{-4}$ & $2 \times 10^{8}$ & $1.5 \times 10^{9}$ & 13.5 & 5 \\
\hline NGC 3628 & $\mathrm{Sbc}$ & 0.16 & $6.7 \times 10^{39}$ & $6 \times 10^{-4}$ & $4 \times 10^{8}$ & $3.8 \times 10^{8}$ & $15 / 25$ & 6 \\
\hline NGC 4565 & $\mathrm{Sb}$ & 0.17 & $2.6 \times 10^{39}$ & $6 \times 10^{-4}$ & $2.2 \times 10^{7}$ & $4.2 \times 10^{8}$ & 13 & 7 \\
\hline $\mathrm{NGC} 4569^{a}$ & $\mathrm{Sab}$ & 0.2 & $1.5 \times 10^{39}$ & $3 \times 10^{-3}$ & $1.5 \times 10^{7}$ & $3 \times 10^{8}$ & 9 & 8 \\
\hline
\end{tabular}

${ }^{a}$ sperical western halo

Column (1): Morphology type;

Column (2): Plasma temperature in units of $\mathrm{keV}$;

Column (3): X-ray luminosity $(0.1-2.4 \mathrm{keV})$ in units of $\operatorname{erg~s}^{-1}$;

Column (4): Mean electron density in units of $\mathrm{cm}^{-3}$;

Column (5): Mass in units of $M_{\odot}$;

Column (6): Cooling time scale in units of yr;

Column (7): Maximal extension in $z$-direction above galactic disk in units of kpc. The two values for NGC 3628 are for the northern and southern halo;

Column (8): References: 1) Strickland et al. (1997), 2) Bomans et al. (1997), 3) Pietsch (1993), 4) Dahlem et al. (1998), 5) Pietsch et al. (1998), 6) Dahlem et al. (1996), 7) Vogler et al. (1996), 8) this work.

Applying the model for a single SNR by Cioffi (1990) and taking into account the cumulative radiation of a number of SNRs in a star-forming region (Norman \& Ikeuchi 1989), we can estimate the SFR from the observed X-ray emission of disk and bulge in NGC 4569 (Junkes et al. 1995). A SNR expanding into an ISM with a mean density of $1 \mathrm{~cm}^{-3}$ radiates a total energy of $4.7 \times 10^{49} \mathrm{ergs}$ in the ROSAT energy band for about $10^{4} \mathrm{yr}$.

With a total X-ray luminosity of $1.8 \times 10^{40} \mathrm{erg} \mathrm{s}^{-1}$ from disk and bulge we derive a number of 120 SNRs and a SNII rate of $0.012 \mathrm{yr}^{-1}$. With a Salpeter IMF between $0.1 M_{\odot}$ and $100 M_{\odot}$ and all stars with masses above $8 M_{\odot}$ evolving to SNeII, we derive a SFR of $1.6 M_{\odot} \mathrm{yr}^{-1}$ for NGC 4569. For comparison, we can estimate the SFR from the FIR luminosity ${ }^{2} L_{\mathrm{FIR}}=5.64 \times 10^{9} L_{\odot}$ (Rice et al. 1988) according to Thronson \& Telesco (1986) to be $3.7 M_{\odot} \mathrm{yr}^{-1}$, what is about 2 times higher than the result from the X-ray luminosity.

\subsection{Interaction with the Virgo cluster gas}

Let us now consider both observational facts, namely, the absence of an extended eastern halo counterpart and the much softer X-ray spectrum of the southern disk (the 0.5$0.9 \mathrm{keV}$ range (Fig. 4c) is quite symmetrical in the disk, whereas the $0.1-0.4 \mathrm{keV}$ image is not). In addition, we address the question whether both effects can be combined to fit into one consistent picture. For this we invoke a onesided external influence, e.g. by the ICM.

The high radial velocity of NGC 4569 relative to the Virgo cluster and the close projected distance of 1.7 toward M 87 suggest that the ICM has a strong influence on the morphology of the galactic gas. Numerical mod-

\footnotetext{
${ }^{2}$ Value adapted for the distance of $16.8 \mathrm{Mpc}$.
}

els of the interaction between galactic ISM and the ICM lead to the result that significant removal of ISM gas from a galaxy penetrating through a cluster medium happens not before the galaxy has already passed its pericenter (Vollmer et al. 2000). On the other hand, the high relative velocity of NGC 4569 with a radial velocity component of about $1250 \mathrm{~km} \mathrm{~s}^{-1}$ to Virgo might indicate a location close to this pericenter. From this fact in combination with its Hi deficiency it seems conclusive that NGC 4569 is not on its first passage through the cluster. Furthermore it penetrates the ICM supersonically. A sound speed of $c_{\mathrm{s}} \approx 500 \mathrm{~km} \mathrm{~s}^{-1}$ (Veilleux et al. 1999) for the ICM leads to a Mach number of at least $M=v / c_{\mathrm{s}} \approx 2.5$. The galaxy and any large-scale outflow from the disk are exposed to the strong ram pressure from the ICM. Even more for the extended halo gas the orientation of the disk with respect to the motion becomes important.

Several indicators (see Bomans et al. 2001) allow to conclude that the disk of NGC 4569 is orientated with its western edge closer to the observer: under the assumption of trailing spiral arms, the Hi velocity field supports this conclusion. Furthermore, the western part exhibits stronger extinction from dust absorption in the western disk. This is also visible in the MIR dust emission image from Roussel et al. (2001). Therefore we conclude that we are looking from east sidewards onto the disk. This is also consistent with the HST $\mathrm{H} \alpha$ observations of the core by Pogge et al. (2000): with enhanced contrast spike-like features are discernible in their Fig. 1c, mainly pointing from the center toward the east. If we now suppose that the outflow of hot gas from the central starburst in NGC 4569 is bipolar (as a working hypothesis), we must assume that the asymmetric X-ray features reflect the existence of a tangential motion of NGC 4569 with respect to the Virgo 
cluster ICM in the sense that the eastern part experiences most of the ram pressure while the western halo is protected and thus undisturbed. Thus the western soft component represents the outflow at the far side of the disk away from the observer, consistent with the radial velocity field of the $\mathrm{H} \alpha$ emission (Bomans et al. 2001).

Since the Hi is concentrated mainly at the center of the galaxy (confirmed by Fig. 17b of Cayatte et al. 1990) the radial extension of the $\mathrm{X}$-ray feature is large enough not to be obscured by the Hi disk. From Fig. 4b and comparing it with Fig. 4c, one can recognize that the southern soft part does not coincide with the disk. It is plausible that the outflow, expanding toward the observer, is compressed by the ICM and bent toward the south. This gives us a clear hint of the tangential $2 \mathrm{~d}$ motion of NGC 4569 to be directed roughly in northeast. Such a protection phenomenon for the western component has been discussed on the basis of a bow shock model by Veilleux et al. (1999) for another Virgo cluster galaxy, NGC 4388, which shows a large-scale ionization cone in $\mathrm{H} \alpha$ from the central Seyfert nucleus. This object has roughly the same high relative velocity to the cluster gas as NGC 4569. Veilleux et al. (1999) argue by the formation of a bow shock, separating the high velocity ICM gas from the slowed-down post-shock gas, with the outflow feature lying in the lee site of the galaxy. The opening angle of the bow shock is determined by the Mach number.

\subsection{Interaction with IC 3583}

The low number of X-ray counts from IC 3583 does not allow a reliable prediction for the nature of the X-ray source in this object. However, the faint southern $\mathrm{H} \alpha$ spur, also traceable in the $B$ band but not in the $R$ band, which therefore may not stem from diffuse HII, indicates ongoing star formation activity. The projected distance of $6^{\prime}$ from NGC 4569 leads to the question as to what extent any interaction between the two objects occurs. One important argument against any perturbation effect is their high relative radial velocity of $1300 \mathrm{~km} \mathrm{~s}^{-1}$. This also makes any detection of connecting features in Hi very difficult, since the velocity range is too large to be covered by the dynamical range of $\mathrm{HI}$ images. A fast passage and the resulting temporary perturbation of the gravitational potential from NGC 4569 may have some influence on the gas component in IC 3583 and may trigger shock-induced star formation. Although no strong tidal feature has been detected in IC 3583 and NGC 4569, some minor distortion like the faint $\mathrm{H} \alpha$ spur may be caused by some weak interaction.

\section{Conclusions}

We have observed the Virgo cluster spiral NGC 4569 and the nearby Magellanic-type irregular galaxy IC 3583 in the optical $R$ and $B$ broad band, in $\mathrm{H} \alpha+[\mathrm{NII}]$, and in the X-ray $0.1-2.4 \mathrm{keV}$ energy band. We have concentrated our analysis on the three regions in NGC 4569: nucleus, halo, and disk. From the absence of a compact X-ray nucleus in the hard ASCA band one can already exclude the existence of an AGN in NGC 4569. Our results also support a central starburst by several facts: The spectral distribution of the nuclear X-ray source favours a thermal plasma emission rather than an AGN-typical power law. The resulting X-ray-to- $\mathrm{H} \alpha$ luminosity ratio of $\log \left(L_{\mathrm{X}} / L_{\mathrm{H} \alpha}\right)=-1.1$ to -0.3 is typical for galaxies with a central starburst and much too low for LINER or Seyfert galaxies. On the other hand, the much higher X-ray luminosity of $1.8 \times 10^{41} \mathrm{erg} \mathrm{s}^{-1}$ for a single power-law model results in an $\mathrm{X}$-ray-to- $\mathrm{H} \alpha$ ratio consistent with other LINERS or Seyferts. Also, the extension of the observed diffuse western soft X-ray component reveals a shape more typical for a galactic wind from a central and/or circumnuclear starburst rather than a more collimated ionisation cone from an AGN.

This soft western X-ray emission is remarkably connected with a very extended $\mathrm{H} \alpha$ filament (Bomans et al. 2001) that reaches from the disk up to at least $9 \mathrm{kpc}$ into the halo of NGC 4569. In combination with its softness the X-ray emission can be interpreted as an X-ray halo fed by the central starburst-driven outflow. Such X-ray halos have by now only been detected in edge-on galaxies, where an easier distinction between sources within and above the galactic disk is possible. NGC 4569 is the first spiral galaxy, where a direct connection of extraplanar $\mathrm{H} \alpha$ and X-ray emitting gas at scales of $10 \mathrm{kpc}$ has been observed. A comparable phenomenon has only been reported by Devine \& Bally (1999) for M 82. The best spectral fit for the X-ray halo in NGC 4569 yields a plasma temperature of $0.2 \mathrm{keV}$ and an X-ray luminosity of $1.5 \times 10^{39} \mathrm{erg} \mathrm{s}^{-1}$. For a particular geometry of the western halo part under the assumption of a $9 \mathrm{kpc}$ halo extension a mean electron density of $3 \times 10^{-3} \mathrm{~cm}^{-3}$ follows, so that its total mass amounts to $1.5 \times 10^{7} M_{\odot}$. The cooling time of $3 \times 10^{8} \mathrm{yrs}$ is long enough to be traceable in X-rays at such a height for an outflow velocity as small as $30 \mathrm{~km} \mathrm{~s}^{-1}$.

One important aspect in obtaining a global picture of NGC 4569 is the spatial orientation of the disk and extraplanar components. From extinction, arm structure and rotation we conclude that the disk is inclined in the sense that the western part is closer. Soft X-ray shadowing by the disk and the reasonable assumption of a perpendicular outflow favour that the western extraplanar features are located behind the galactic disk. The extension of the HI disk is small enough not to hide the western X-ray component. Distortion of X-ray features, like the southern soft emission, and in $\mathrm{H} \alpha$ provoke a dynamical interpretation of a strong interaction with the Virgo ICM at a high relative velocity of at least $1250 \mathrm{~km} \mathrm{~s}^{-1}$.

The Magellanic irregular galaxy IC 3583 contains an unresolved X-ray source which is suggested not to originate from a foreground star present in the optical because of a very hard spectrum. A narrow $\mathrm{H} \alpha$ spur pointing from the southern end toward NGC 4569, also visible in the $B$ band, indicates ongoing star formation in IC 3583. The question of an interaction between the two galaxies at very 
high relative radial velocity of about $1300 \mathrm{~km} \mathrm{~s}^{-1}$ has to be addressed to and must be explored by more detailed observational and model analyses.

Acknowledgements. The authors are grateful for helpful discussions with $\mathrm{Ch}$. Theis and A. Boselli. The paper benefits from constructive remarks by an unknown referee. The ROSAT project is supported by the German Bundesministerium für Bildung, Wissenschaft, Forschung und Technologie (BMBF) and the Max-Planck-Society. This research has made use of the NASA/IPAC Extragalactic Database (NED) which is operated by the Jet Propulsion Laboratory, Caltech, under contract with the NASA. This work was partly supported by the Deutsche Forschungsgemeinschaft under grant No. He 1487/23-1 (D.T.).

\section{References}

Arnaud, K. A. 1996, Astronomical Data Analysis Software and Systems V, ed. G. Jacoby, \& J. Barnes, ASP Conf. Ser., 101,17

Balsara, D. S., Livio, M., \& O’Dea, C. P. 1994, ApJ, 437, 83

Barth, A. J., Ho, L. C., Filippenko, A. V., \& Sargent, W. L. W. 1998, ApJ, 496, 133

Barth, A. J., Ho, L. C., \& Shields, J. C. 2000, PASP, 112, 753

Binggeli, B., Sandage, A., \& Tammann, G. A. 1985, AJ, 90, 1681

Böhringer, H., Briel, U. G., Schwarz, R. A., et al. 1994, Nature, 368,828

Bomans, D. J., Chu, Y.-H., \& Hopp, U. 1997, AJ, 113, 1678

Bomans, D. J., Tschöke, D., Hensler, G., \& Boselli, A. 2001, A\&A, submitted

Bravo-Alfaro, H., Cayatte, V., van Gorkom, J. H., \& Balkowski, C. 2000, AJ, 119,580

Cayatte, V., Kotanyi, C., Balkowski, C., \& van Gorkom, J. H. 1994, AJ, 107, 1003

Cayatte, V., van Gorkom, J. H., Balkowski, C., \& Kotanyi, C. 1990, AJ, 100, 604

Cioffi, D. 1990, in Physical Processes in Hot Plasmas, ed. W. Brinkmann, A. C. Fabian, \& F. Giovanelli (Kluwer, Dordrecht), NATO ASI Ser. C, 305, 1

Colina, L., \& Arribas, S. 1999, ApJ, 514, 637

Dahlem, M. 1997, PASP, 109, 1298

Dahlem, M., Heckman, T. M., Fabbiano, G., Lehnert, M. D., \& Gilmore, D. 1996, ApJ, 461, 724

Dahlem, M., Petr, M. G., Lehnert, M. D., Heckman, T. M., \& Ehle, M. 1997, A\&A, 320, 731

Dahlem, M., Weaver, K. A., \& Heckman, T. M. 1998, ApJS, 118,401

Devine, D., \& Bally, J. 1999, ApJ, 510, 197

Dickey, J. M. 1997, AJ, 113, 1939

Dickey, J. M., \& Lockman, F. J. 1990, ARA\&A, 28, 215

Fabbiano, G., Kim, D.-W., \& Trinchieri, G. 1992, ApJS, 80, 531

Falco, E., Kurtz, M., Gellar, M., et al. 1999, PASP, 111, 438

Freedman, W. L. 1996, in Critical Dialogs in Cosmology, ed. N. Turok (World Scientific Publishing), 92

Garcia, A. M. 1993, A\&AS, 100, 47

Heckman, T. M., Lehnert, M. D., \& Armus, L. 1993, in The Environment and Evolution of Galaxies, ed. J. M. Shull, \& H. R. Thronson, Jr. (Kluwer Academic Publishers, Dordrecht), 455
Hensler, G., Dickow, R., Junkes, N., \& Gallagher, J. S. 1998, ApJ, 502, L17

Hunter, D. A., \& Gallagher, J. S. 1990, ApJ, 362, 480

Junkes, N., Zinnecker, H., Hensler, G., Dahlem, M., \& Pietsch, W. 1995, A\&A, 294, 8

Junkes, N., \& Hensler, G. 1996, in The Interplay between Massive Star Formation, the ISM and Galaxy Evolution, 11th IAP Meeting, ed. D. Kunth, et al., 533

Keel, W. C. 1983, ApJ, 269, 466

Keel, W. C. 1996, PASP, 108, 917

Kennicutt, R. C., Jr., Tamblyn, P., \& Congdon, C. E. 1994 ApJ, 435, 22

Lehnert, M. D., Heckman, T. M., \& Weaver, K. A. 1999, ApJ, 523,575

Maoz, D., Filippenko, A. V., Ho, L. C., et al. 1995, ApJ, 440, 91

Neff, S. G., \& Hutchings, J. B. 1992, AJ, 103, 1746

Norman, C. A., \& Ikeuchi, S. 1989, ApJ, 345, 372

Pérez-Olea, D. E., \& Colina, L. 1996, ApJ, 468, 191

Pietsch, W. 1993, in Panchromatic View of Galaxies - Their Evolutionary Puzzle, ed. G. Hensler, Ch. Theis, \& J. S. Gallagher, 137

Pietsch, W., Trinchieri, G., \& Vogler, A. 1998, A\&A, 340, 351

Pogge, R. W., Maoz, D., Ho, L. C., \& Eracleous, M. 2000, ApJ, 532,323

Raymond, J. C., \& Smith, B. W. 1977, ApJS, 35, 419

Rice, W., Lonsdale, J. C., Soifer, B. T., et al. 1988, ApJS, 68, 91

Roussel, H., Vigroux, L., Bosma, A., et al. 2001, A\&A, 369, 473

Saikia, D. J., Unger, S. W., Pedlar, A., et al. 1990, MNRAS, 245,397

Sandage, A., \& Bedke, J. 1985, AJ, 90, 2006

Sérsic, J. L. 1973, PASP, 85, 103

Shopbell, P. L., \& Bland-Hawthorn, J. 1998, ApJ, 493, 129

Stauffer, J. R. 1983, ApJ, 264, 14

Stauffer, J. R., Kenney, J. D., \& Young, J. S. 1986, AJ, 91, 1286

Strickland, D. K., Heckman, T. M., Weaver, K. A., \& Dahlem, M. 2000, AJ, 120, 2965

Strickland, D. K., Ponman, T. J., \& Stevens, I. R. 1997, A\&A, 320,378

Suchkov, A. A., Balsara, D. S., Heckman, T. M., \& Leitherer, C. 1994, ApJ, 430, 511

Tanvir, N. R., Shank, T., \& Major, J. V. 1991, MNRAS, 253, $21 \mathrm{p}$

Terashima, Y., Ho, L. C., Ptak, A. F., et al. 2000, ApJ, 533, 729

Thronson, H. A., \& Telesco, C. M. 1986, ApJ, 311, 98

Tschöke, D., Hensler, G., \& Junkes, N. 1999, A\&A, 343, 373

Tschöke, D., Hensler, G., \& Junkes, N. 2000, A\&A, 360, 447

Tschöke, D., Hensler, G., \& Junkes, N. 2001, A\&A, submitted

Tully, R. B. 1988, Nearby Galaxies Catalog (Cambridge University Press, Cambridge)

Veilleux, S., Bland-Hawthorn, J., Cecil, G., Tully, R. B., \& Miller, S. T. 1999, ApJ, 520, 111

Vogler, A., Pietsch, W., \& Kahabka, P. 1996, A\&A, 305, 74

Vollmer, B., Marcelin, M., Amram, P., et al. 2000, A\&A, 364, 532

Warmels, R. H. 1988, A\&AS, 72, 19

Yasuda, N., Fukugita, M., \& Okamura, S. 1997, ApJS, 108, 417

Zimmermann, H. U., Belloni, T., Izzo, C., Kahabka, P., \& Schwentker, O. 1993, EXSAS User's Guide, Edition 3, ROSAT Scientific Data Center (Garching) 